\title{
Genome-wide analysis of basic helix-loop-helix transcription factors in papaya (Carica papaya $L_{\text {. }}$ )
}

\author{
Min Yang ${ }^{1}$, Chenping Zhou ${ }^{1}$, Hu Yang ${ }^{1}$, Ruibin Kuang ${ }^{1}$, Bingxiong Huang ${ }^{1}$, Yuerong Wei ${ }^{\text {Corresp. } 1}$ \\ ${ }^{1}$ Guangdong Academy of Agricultural Sciences, Key Laboratory of South Subtropical Fruit Biology and Genetic Resource Utilization (MOA), Guangdong \\ Province Key Laboratory of Tropical and Subtropical Fruit Tree Research, Institute of Fruit Tree Research, Guangzhou, China \\ Corresponding Author: Yuerong Wei \\ Email address: weiyuerong@gdaas.cn
}

The basic helix-loop-helix (bHLH) transcription factors (TFs) have been identified and functionally characterized in many plants. However, no comprehensive analysis of the bHLH family in papaya ( Carica papaya L.) has been reported previously. Here, a total of 73 CpbHLHs were identified in papaya, and these genes were classified into 18 subfamilies based on phylogenetic analysis. Almost all of the CpbHLHs in the same subfamily shared similar gene structures and protein motifs according to analysis of exon/intron organizations and motif compositions. The number of exons in CpbHLHs varied from 1 to 10 with an average of 5 . The amino acid sequences of the bHLH domains were quite conservative, especially Leu-27 and Leu-63. Promoter cis-element analysis revealed that most of the $\mathrm{CpbHLHs}$ contained cis-elements that can respond to various biotic/abiotic stress-related events. Gene ontology (GO) analysis revealed that CpbHLHs mainly functions in protein dimerization activity and DNA-binding, and most CpbHLHs were predicted to localize in the nucleus. Abiotic stress treatment and quantitative real-time PCR (qRT-PCR) revealed some important candidate $\mathrm{CpbHLHs}$ that might be responsible for abiotic stress responses in papaya. These findings would lay a foundation for further investigate of the molecular functions of CpbHLHs. 
2 Genome-wide analysis of basic helix-loop-helix

3 transcription factors in papaya (Carica papaya L.)

4

5

6

7

8

Key Laboratory of South Subtropical Fruit Biology and Genetic Resource Utilization (MOA),

9 Guangdong Province Key Laboratory of Tropical and Subtropical Fruit Tree Research, Institute

Corresponding Author:

Yuerong Wei

80 Dafeng 2nd Street, Huijing North Road, Tianhe District, Guangzhou, Guangdong, 510640, China

Email address: weiyuerong@gdaas.cn 


\section{Abstract}

43 The basic helix-loop-helix (bHLH) transcription factors (TFs) have been identified and

44

45

46

47

48

49

50

51

52

53

54

55

56

57

58

59

60

61

62

63

64

65

66

67

68

69

70

71

72

73

74

75

76

77

78

79

80

81

82

functionally characterized in many plants. However, no comprehensive analysis of the bHLH family in papaya (Carica papaya L.) has been reported previously. Here, a total of 73 CpbHLHs were identified in papaya, and these genes were classified into 18 subfamilies based on phylogenetic analysis. Almost all of the CpbHLHs in the same subfamily shared similar gene structures and protein motifs according to analysis of exon/intron organizations and motif compositions. The number of exons in CpbHLHs varied from 1 to 10 with an average of 5. The amino acid sequences of the bHLH domains were quite conservative, especially Leu-27 and Leu63. Promoter cis-element analysis revealed that most of the CpbHLHs contained cis-elements that can respond to various biotic/abiotic stress-related events. Gene ontology (GO) analysis revealed that $\mathrm{Cp} b H L H s$ mainly functions in protein dimerization activity and DNA-binding, and most $\mathrm{CpbHLHs}$ were predicted to localize in the nucleus. Abiotic stress treatment and quantitative real-time PCR (qRT-PCR) revealed some important candidate CpbHLHs that might be responsible for abiotic stress responses in papaya. These findings would lay a foundation for further investigate of the molecular functions of CpbHLHs.

\section{Introduction}

Since plants are unable to move, plant growth and development are regularly affected by abiotic and biotic stresses, which impair yields and result in losses to farmers. For better growth and development, plants have to make use of a series of physiological and biochemical processes in their responses to multiple abiotic stresses by regulating gene expression (Agarwal et al. 2006; Feller et al. 2011; Pires \& Dolan 2010). And previous studies have demonstrated that these physiological and biochemical mechanisms are more likely to be a polygenic cooperative defense response induced by various stresses, rather than the single response of a single gene (Zhang et al. 2004). Therefore, the traditional method of obtaining the stress tolerance of plants by modification a single resistance/sensitive gene is limited. Comprehensive analysis of important gene families are very important for molecular breeding. As an important and popular fruit, papaya is famous for its high nutritional and medical values. Papaya is widely grown in southern China, the tropics and subtropics areas, and its demand is increasing every year. However, the production and quality of papaya were often threatened by various abiotic stresses, such as salt, drought, and cold. These stresses often cause severe economic losses in papaya production in China. So it is very important to study the functions of gene families that involved in abiotic stresses response in papaya. Since obtaining the whole genome sequences of papaya (Ming et al. 2008), several important gene families have been identified by the tool of genome-wide analysis in papaya, including Aux/IAA gene family, ARF family, SQUAMOSA promoter binding protein-like $(S P L)$ gene family, NBS resistance gene family and NPR1 family. These families were essential for papaya fruit ripening, flower and fruit development, fitness and disease resistance (Liu et al. 2017a; Liu et al. 2015; Peraza-Echeverria et al. 2012; Porter et al. 2009; Xu et al. 2020).

Peer) reviewing PDF | (2020:02:45453:1:0:CHECK 15 Apr 2020) 
83 In various stresses regulation network and signaling pathways, transcription factors (TFs) are a

84 kind of important proteins that regulate gene expression by activating and repressing related

85 downstream genes. Among them, WRKY and bHLH families are the most common TF families

86 in higher plants (Kosugi \& Ohashi 2002). And the WRKY transcription factors has been

87 reported to be related to abiotic and biotic stresses responses in papaya (Pan \& Jiang 2014).

$88 \mathrm{Basic} /$ helix-loop-helix (bHLH) TFs are widely found in almost all eukaryotes and are the second

89 largest TFs family in plants (Carretero-Paulet et al. 2010; Feller et al. 2011; Jones 2004; Pires \&

90 Dolan 2010). The bHLH superfamily proteins are defined by one highly conserved bHLH

91 domain, which comprises approximately 60 amino acids in length and contains two different

92 functional regions: the basic region and the HLH region (Li et al. 2006; Toledo-Ortiz et al.

93 2003). The basic region is located at the N-terminal end of the bHLH domain and consists

94 approximately 15 amino acids. It is a DNA-binding region that enables bHLH TFs to bind to a

95 specific E-boxes (CANNTG) (Atchley \&Fitch 1997; Atchley et al. 1999). The HLH region, at

96 the C-terminal end, is mainly composed of hydrophobic residues, containing two amphipathic $\alpha$ -

97 helices linked by a loop region that has variable sequences and acts as a dimerization domain

98 (Heim et al. 2003; Li et al. 2006). Outside of the two conserved regions, the rest of the bHLH

99 protein sequences are usually very different (Morgenstern \& Atchley 1999).

100 In animals, the bHLH TFs can be divided into six main groups (designated A to F) based on

101 phylogenetic analysis, functional properties and DNA-binding specificity (Atchley \&Fitch

102 1997). These bHLH groups can be divided into several small subfamilies (Ledent \& Vervoort

103 2001; Simionato et al. 2007). The bHLHs mainly function in sensing the external environment,

104 cell cycle regulation and tissue differentiation (Amoutzias et al. 2004; Atchley \& Fitch 1997;

105 Stevens et al. 2008; Vervoort \& Ledent 2001). Compared to animals, the research on bHLH

106 proteins in plants is limited, even the exact number subfamilies of bHLH TFs has not been

107 determined. Generally, the bHLH proteins is thought to cover 15-25 subfamilies (Buck \&

108 Atchley 2003; Pires \& Dolan 2010), but some atypical bHLHs have extended the number to 32

109 based on phylogenetic analysis in plants (Carretero-Paulet et al. 2010). With the availability of

110 genome sequence data and the rapid development of molecular biology, increasing numbers of

111 bHLH subfamily genes have been identified and characterized in a wide range of plant species,

112 including Arabidopsis (Toledo-Ortiz et al. 2003), peanut (Gao et al. 2017), apple (Mao et al.

113 2017), tomato (Sun et al. 2015), potato (Wang et al. 2018b), peach (Zhang et al. 2018), grapes

114 (Wang et al. 2018a), sweet orange (Geng \& Liu 2018), and bamboo (Cheng et al. 2018). The

115 results from these research have shown that bHLH TFs have versatile biological functions, such

116 as regulating light morphogenesis (Leivar et al. 2008; Roig-Villanova et al.2007), hormone

117 signals (Friedrichsen et al. 2002; Lee et al. 2006), the developmental of root (Feng et al. 2017)

118 and anther (Farquharson 2016), regulating epidermal cell fate determination (Bernhardt et al.

119 2003), participating in various biotic and abiotic stress responses (Jiang et al. 2009; Liu et al.

120 2014; Wang et al. 2018b), etc.

121 In recent years, some studies demonstrated that bHLH transcription factors play important roles

122 in the stress-related regulation network and signaling pathways in many species. However, no

PeerJ reviewing PDF | (2020:02:45453:1:0:CHECK 15 Apr 2020) 
123

124

125

126

127

128

129

130

131

132

133

134

135

136

137

138

139

140

141

142

143

144

145

146

147

148

149

150

151

152

153

154

155

156

157

158

159

160

161

162

systematic analysis of the bHLH TFs have previously been performed in papaya. In this study, a total of $73 \mathrm{CpbHLH}$ genes were identified in papaya, and phylogenetic analyses were carried out to analyze the relationships among these genes. Meanwhile, gene structure, protein physicochemical properties and conserved motifs, the cis-element of the promoter region, and gene ontology (GO) analysis were investigated. Furthermore, to analyze the functions of $C p b H L H s$ responsible for responding to abiotic stresses, the expression profiles of 22 selected genes under salt, drought, ABA and cold stresses were investigated by using quantitative realtime PCR (qRT-PCR). We identified several important candidate genes that might be responsible for abiotic stress responses. We completed the first comprehensive genome-wide analysis of the bHLH gene family in papaya, and our results provide information necessary for further functional research of the bHLH family in papaya.

\section{Materials \& Methods}

\section{Identification of $\mathbf{C p b H L H}$ genes, gene structure and physicochemical analysis}

Papaya (Carica papaya L.) bHLH protein sequences were downloaded from the Plant TFDB V4.0 database (Jin et al. 2017). Furthermore, we used the SMART online software (http:// smart.embl-heidelberg.de/) and the InterProScan tool (http://www.ebi.ac.uk/Tools/pfa/iprscan/) to identify integrated bHLH domains in putative papaya bHLH proteins. The physicochemical properties of $C p b H L H$ proteins were predicted by ProPAS (Wu \& Zhu 2012). The genomic sequences, ID numbers and coding sequences (CDS) corresponding to each predicted CpbHLH gene were obtained from the Phytozome database (https://phytozome.jgi.doe.gov/pz/portal.html). The intron numbers, exon-intron organizations and locations of the $C p b H L H$ genes were analyzed by Gene Structure Display Server (GSDS) v2.0 (Hu et al. 2015).

\section{Phylogenetic tree building, motif identification and multiple sequence alignment}

To research the phylogenetic relationship of $C p b H L H$ proteins, protein sequences of papaya were pre-aligned using HMM align (Eddy 1998) and the pHMM HLH ls.hmm from PFAM (https://pfam.xfam.org/family/PF00010) to identify the domains of bHLH TFs. Based on the manually aligned bHLH region of $158 \mathrm{bHLH}$ proteins from Arabidopsis and 173 from rice (Pires \& Dolan 2010), the identified bHLH domains were later aligned using MAFFT v7.305b (Kaotoh et al. 2002) with default settings. Phylogenetic tree was constructed based on the neighborjoining method using FastTree v2.1.11 (Price et al. 2009) with default settings. Bootstrapping with 1000 replicates was used to assess the statistical reliability of nodes in the tree. Multiple sequence alignment based on protein sequences of these $73 \mathrm{CpbHLH} \mathrm{TFs} \mathrm{was} \mathrm{generated} \mathrm{by}$ MAFFT v7.305b (Kaotoh et al. 2002) with default settings.

To identify the conserved motifs among the $C p b H L H$ proteins, we uploaded the 73 amino acid sequences of the $C p b H L H$ family to the Multiple EM for Motif Elicitation (MEME, version 5.02) (http://meme-suite.org/tools/ meme). The parameter settings were as follows: zero or one, occurrence of a single motif per sequence; 3, maximum number of motifs found. All other parameters were set to the default values.

Promoter cis-acting Regulatory Element Analysis and Gene Ontology (GO) Annotation 
163 To predict and compare the putative promoter cis-elements of bHLHs in papaya and Arabidopsis,

164

165

166

167

168

169

170

171

172

173

174

175

176

177

178

179

180

181

182

183

184

185

186

187

188

189

190

191

192

193

194

195

196

197

198

199

200

201

202

the upstream 2000 bp genomic DNA sequences of 73 CpbHLH genes in papaya, and 47 AtbHLH genes in Arabidopsis (the putative orthologous genes corresponding to CpbHLHs) were downloaded and then submitted to the PlantCARE (Magali et al. 2002). The full-length protein sequences of papaya bHLH were blasted against Arabidopsis proteins with default parameters. The best hits were submitted to AgriGOv2.0 (http://systemsbiology.cau.edu.cn/ agriGOv2/) for GO annotation (Tian et al. 2017). GO terms include three aspects: biological process, cellular component and molecular function.

\section{Plant materials, growth conditions and stress treatments}

In this experiment, stems with axillary buds were selected as explants from two-year-old 'Yi Chi Gua' papaya trees grown under standard field conditions in the Institute of Fruit Tree Research, Guangdong Academy of Agriculture Science, Guangzhou, China, and cultured in vitro to obtain the complete papaya seedlings with normal leaves and roots using tissue culture techniques. Healthy and uniform papaya seedlings were used for different treatments. For the selection of stress conditions for papaya, we designed different gradients of stress conditions for pre-experiments: the concentration gradients of salt stress are $100 \mathrm{mM} \mathrm{Nacl}, 200 \mathrm{mM} \mathrm{Nacl}$, and $300 \mathrm{mM} \mathrm{Nacl}$; the concentration gradients of PEG6000 (to mimic drought stress) are 15\% PEG6000, 20\% PEG6000, 25\% PEG6000 and 30\% PEG6000; the concentration gradients of $\mathrm{ABA}$ are $50 \mu \mathrm{M}$ ABA, $100 \mu \mathrm{M} \mathrm{ABA}$ and $150 \mu \mathrm{M}$ ABA; the temperature gradients are $0{ }^{\circ} \mathrm{C}, 4^{\circ} \mathrm{C}$ and $10^{\circ} \mathrm{C}$, and finally determined the suitable stress conditions used in this manuscript. For salt, drought and ABA stresses, seedlings were treated with MS liquid medium containing $200 \mathrm{mM}$ Nacl, 25\% PEG6000 and $100 \mu \mathrm{M}$ ABA for 2 hours respectively, and then the roots were collected. For cold treatment, seedlings were subjected to $4^{\circ} \mathrm{C}$ for 2 hours and the leaves were collected. All of the collected materials were immediately frozen in liquid nitrogen and stored at $-80^{\circ} \mathrm{C}$ for RNA isolation. Untreated seedlings were used as the control groups. Three biological replications were carried out for each treatment.

\section{RNA extraction and quantitative real-time PCR (qRT-PCR) analysis}

Total RNA from papaya after different treatments was isolated using TRIzol reagent (Invitrogen). The extracted RNA was treatment with DNase (TaKaRa), and then reverse transcribed into cDNA using the PrimeScriptTM RT Reagent Kit (TaKaRa). The qRT-PCR was conducted on the ABI StepOne Real Time PCR system using 2X SG Fast qPCR Master Mix (High Rox) (TaKaRa) according to the manufacturer's instructions. TATA binding protein 2 (CpTBP2) amplification was used as an internal control (Zhu et al. 2012). The qRT-PCR reactions used three biological replicates, and each biological repeat had three technical replicates. Gene-specific primers for qRT-PCR of the $22 \mathrm{CpbHLH}$ genes were designed based on the CDSs of the CpbHLH genes using Primer Premier 5.0 (Data S1). The relative expression levels of each gene were calculated using the $2^{-\Delta \Delta C T}$ method, the raw data was showed in supplemental file 1 .

\section{Results}




\section{Identification and characterization of CpbHLHs}

204 A total of 105 putative bHLH transcription factors of papaya (C. papaya) were downloaded from 205 the PlantTFDBv2.0 (http://planttfdb.cbi.pku.edu.cn/). To verify the reliability of these results, the $206105 \mathrm{CpbHLH}$ proteins sequences were filtered by Interproscan and SMART domain annotation, 207 and a total of 73 predicted $C p b H L H$ proteins were identified. They were named CpbHLH001 to $208 C p b H L H 073$ at random except for 32 proteins that were explicitly excluded by Interproscan and 209 SMART (Table S1). The detailed information on these predicted CpbHLHs, including protein 210 ID, locus ID, opening reading frame (ORF) lengths, amino acid sequences/lengths, molecular 211 weight, isoelectric point and exon/intron numbers, are listed in Data S2. In previous studies, 212 129/132, 188, 159, 147, 124, 95, 94 and 56 bHLH genes were identified in peanut (Gao et al. 213 2017), apple (Mao et al. 2017), tomato (Sun et al. 2015), Arabidopsis (Toledo-Ortiz et al. 2003), 214 potato (Wang et al. 2018b), peach (Zhang et al. 2018), grapes (Wang et al. 2018a) and sweet 215 orange (Geng \& Liu 2018), respectively. Compared with the above dicotyledonous plants, the 216 density of $b H L H s$ genes in papaya genome was about $0.26 \%$, which is lower than the density of 217 peanut (Gao et al. 2017), apple (Mao et al. 2017), tomato (Sun et al. 2015), Arabidopsis

218 (Toledo-Ortizet al. 2003), potato (Wang et al. 2018b) and sweet orange (Geng \& Liu 2018), and 219 similar to peach (Zhang et al. 2018) and wine grapes (Wang et al. 2018a) (Table 1). This is

220

221

222

223

224

225

226

227

228

229

230

231

232

233

234

235

236

237

238

239

240

241

242 probably associated with the whole-genome duplications during evolution. Among the above plants, some plants with recent whole-gene duplication like peanut, apple, tomato, Arabidopsis, potato and sweet orange while the plants without whole-gene duplication like papaya, peach and wine grapes.

To further characterize the bHLHs in papaya, the physicochemical properties of these putative proteins were analyzed and are shown in Data S2. The size of deduced CpbHLHs ranged from 100 ( $C p$ bHLH053) to 679 ( $C p$ bHLH068) amino acids, the corresponding molecular weights from $11.525 \mathrm{KDa}$ to $75.899 \mathrm{KDa}$. The predicted theoretical isoelectric points (PI) values of CpbHLHs were between 4.71(CpbHLH028) and 11.07(CpbHLH003). Similar molecular weights and isoelectric points have been made in potato (Wang et al. 2018b). And all of predicted CpbHLH proteins were hydrophilic characteristic proteins, the grand average of hydropathy values were negative, ranging from -0.2098( $C p b H L H 033)$ to -1.0125(CpbHLH006). Similar result has been made in Brachypodium distachyon (Niu et al. 2017). That is, the predicted $C p b H L H$ proteins showed diversities in their length, molecular weight, PI and the grand average of hydropathy values.

\section{Phylogenetic analysis, gene structure, conserved motifs analysis and multiple sequence alignment of $\boldsymbol{C p b H L H s}$}

To evaluate the evolutionary relationships of the $C p b H L H$ proteins, a neighbor-joining phylogenetic tree was generated using conserved bHLH domains from papaya, Arabidopsis and rice. The phylogenetic tree showed that the $73 \mathrm{CpbHLH}$ members were clustered into 18 subfamilies with one orphan (Fig.1A and Data S3), consistent with the earlier results showing that the bHLH subfamily in plants can be divided into 15-25 subfamilies (Pires \& Dolan 2010). Previous research have named the bHLH subfamilies using English letters (Li et al. 2006; Mao et 
243 al. 2017), Roman numerals (Song et al. 2017; Sun et al. 2015), or Arabic numerals (Chen et al. 244 2015; Toledo-Ortiz et al. 2003), In this study, we named CpbHLH subfamilies using Roman 245 numerals. As shown in figure 1, the subfamily XII was the largest subfamily among all three 246 species, and all of subfamilies include at least two species. In papaya, none of the bHLHs were 247 grouped into IVd, II, X V, X, XI V and X III subfamilies compared to rice and Arabidopsis, 248 which may be due to these bHLHs were lost during the process of evolution.

249 Exon/intron organization, as a type of structural divergence, plays an important role in the

250

251

252

253

254

255

256

257

258

259

260

261

262

263

264

265

266

267

268

269

270

271

272

273

274

275

276

277

278

279

280

281 evolution of multiple gene families (Xu et al. 2012). The annotation features of the CpbHLH genes were submitted to Gene Structure Display Server (GSDS) together to show their gene structures. As described in Data S2 and Figure 2A, the number of introns varied from zero to ten, representing a complex distribution pattern. Most (63 (86.3\%)) of the CpbHLHs were found to possess introns among the $73 C p b H L H$ genes, while 10 (13.7\%) of the genes were intron-less, 8 $(11.0 \%)$ genes contained one intron, and the remaining genes had two or more introns. In addition, members of the same subfamily also displayed similar intron distribution patterns in view of the full-length genome sequences. For instance, all of the CpbHLHs in subfamily Vb had one intron and two exons, the whole members of subfamily IIIf had six introns and seven exons, the IVa subfamily members showed three introns and four exons, and all members of VIllb subfamily consisted only one exon.

Most importantly, members of the same bHLHs subfamily are usually participated in the same signaling pathway or biological process, and the functions of these members are often partially or totally redundant (Pires \& Dolan 2010). For example, AtbHLH10, AtbHLH89 and AtbHLH91, corresponding rice orthologs OsbHLH141, OsbHLH142 are members of subfamily II, they are all involved in the process of pollen development ( $\mathrm{Li}$ et al. 2006; Liu et al. 2017; Zhu et al. 2015). Especially in Arabidopsis, there is no obvious phenotype in single mutant of AtbHLH10, AtbHLH89 or AtbHLH91, only their various double or triple mutants showed the phenotype of pollen development deficiency (Liu et al. 2017). In subfamily IIIb, OsbHLH001 (OsICE2), OsbHLH002 (OsICE1), CpbHLH027, CpbHLH062, AtbHLH116 (ICE1) and AtbHLH33 (ICE2) were clustered within one clade. In previous studies, AtbHLH116(ICE1) and AtbHLH33(ICE2) and corresponding orthologs in rice (OsbHLH001/OsICE2, OsbHLH002/OsICE1) have been reported to function in the stress of chilling (Chinnusamy et al. 2003; Deng et al. 2017; Fursova et al. 2009; Li et al. 2010; Zhang et al. 2017). And we also found transcripts of CpbHLH027 and CpbHLH062 were increased under chilling stress in this study, implying that $C p$ bHLH027 and CpbHLH062 are involved in the process of chilling stress in papaya.

To further study the sequence characteristics of the predicted bHLH domains at the amino acid level, we carried out a multiple sequence alignment of the 73 predicted $C p b H L H$ protein sequences (Fig.3). The result showed that the 73 putative $C p b H L H$ proteins contained two conserved regions in the bHLH domains: the basic region plus helix 1 and the loop region plus helix 2 (Fig. 3 and Table S2). Additionally, we used the online MEME program to identify the conserved motifs (Bailey \& Elkan 1994). The result also showed that most of the sequences 
282 (exclude $C p$ bHLH003) exhibited two highly conserved motifs: one is contains 29 amino acids, 283 and the other consists of 21 amino acids, are shown in red and blue blocks, respectively (Fig.2B). 284 Among the two motifs, motif 1 comprises basic residues and helix 1, and motif 2 comprises a 285 loop and helix 2. And the space between motif 1 and 2 consists of a loop, which is variable in 286 length in some bHLH proteins. The sequence logos of motif 1 (in red) and motif 2 (in blue) are 287 shown in Figure 4A. The backbones of motif 1 and 2 are also conserved in most plant species 288 (Guo \& Wang 2017; Heim et al. 2003; Sun et al. 2015), and these highly conserved residues in 289 bHLH domains may be responsible for protein dimerization (Heim et al. 2003).

290 Besides these two common conserved motifs, some CpbHLHs that are mainly distributed into

291

292

293

294

295

296

297

298

299

300

301

302

303

304

305

306

307

308

309

310

311

312

313

314

315

316

317

318

319

320

321 eight subfamilies (including $\mathrm{V}$ a, V b, IIIf, IVa, IIIb, III, I b and I a subfamilies) harbor another conserved motif (motif 3 ) with a length of 36 amino acids. The motif 3 is indicated by the green blocks and the sequence logo is visualized as logo3 (Fig. 2B and Fig. 4B). This result is accord with the previous studies that members of a given subfamily exhibited another conserved nonbHLH motif (motif 3) in plant bHLH superfamily (Pires \& Dolan 2010). However, in papaya, members of the bHLH proteins have the same motif that is distributed into eight subfamilies, not just one subfamily. In addition, among the $73 \mathrm{CpbHLHs}$, one atypical bHLH protein (CpbHLH003) exhibited incomplete bHLH domains, whereas the remaining $72 \mathrm{CpbHLH}$ proteins all presented complete bHLH domains. Similar observations have been made in other plant species, such as peach and blueberry (Song et al. 2017; Zhang et al. 2018).

\section{Promoter analysis of $\boldsymbol{b H L H}$ genes in papaya}

To further understand $C p b H L H s$ functions and regulation patterns, cis-elements in $C p b H L H$ genes promoter sequences were investigated. Regions of 2,000 bp upstream from the start codons of each $C P b H L H$ gene were analyzed using PlantCARE. The results showed that the ciselements could be divided into three main categories (Fig. 5A and Data S4). Category one contained a ubiquitous class of plant light responsive elements among which G-Box, G-box, GT1-motif and Box 4 were common in the CpbHLH promoters. Category two contained important elements that were involved in the process of stress-responsiveness, including MYB binding site involved in drought-inducibility (MBS), low temperature response elements (LTR), defense and stress responsive elements (TC-rich) and wound-responsive elements (WUNmotifs). In addition, more than ten kinds of hormone-responsive cis-elements were identified (e.g., gibberellin-GA, auxin-IAA, methyl jasmonate-MeJA, salicylic acid-SA, and abscisic acidABA). Among them, the most common response elements were ABA (ABRE), MeJA (CGTCAmotif and TGACG-motif) and SA (TCA-element and SARE), which included $158(29.15 \%), 128$ $(23.62 \%)$ and $53(9.78 \%)$, respectively (Fig. 5B).Category three contained plant growth and development elements, such as anaerobic induction elements (ARE), $\mathrm{O}_{2}$-site, CAT-box and so on. Additionally, we also analyze the cis-elements in the promoter regions of putative orthologous genes that corresponding to CpbHLH in Arabidopsis, and the similar result has been obtained in Arabidopsis (Fig. S1 and Data S4). There also existed three main categories: plant light, abiotic and biotic stresses and plant growth and development responsive elements. And the percentage of most stress-responsive elements in Arabidopsia were similar to papaya, including 
322

323

324

325

326

327

328

329

330

331

332

333

334

335

336

337

338

339

340

341

342

343

344

345

346

347

348

349

350

351

352

353

354

355

356

357

358

359

360

361

ABA responsive elements, drought-responsive elements, wound-responsive elements, low temperature-responsive elements and IAA responsive elements, implying that most of the promoter cis-elements of bHLH family were conserved in Arabidopsis and papaya.

\section{GO annotation of $C p b H L H$ proteins}

To understand the functions of papaya bHLHs, we performed a GO annotation of $C p b H L H s$, and the results are shown in Data S5. A total of $70 \mathrm{CpbHLHs}$ were involved in protein dimerization activity (GO: 0046983). The result is consistent with the earlier studies, which show that the HLH domain was necessary for protein dimerization and DNA binding (Murre et al. 1989). Some conserved amino acid residues are important to the function of bHLH proteins, especially the Leu-27 in helix 1 and the Leu-73 in helix 2 (Carretero-Paulet et al. 2010). In this study, we found 72 (out of 73) CpbHLH proteins have Leu-27 (corresponding to Leu-27 in AtbHLHs), and all of the $C p$ bHLH proteins have Leu-63 (corresponding to Leu-73 in AtbHLHs) (Fig. 3 and Table S2).

Because of a lack of reported experimental data and databases, we used Arabidopsis as the reference species to perform a GO annotation of $C p b H L H$ proteins, and 54 of 73 predicted CpbHLH proteins were obtained with results compared to Arabidopsis. We summarized the results in Figure 6 and Data S6. The majority of predicted $C p b H L H$ proteins were involved in DNA binding. Almost all of the predicted $C p b H L H$ proteins $(37,68.5 \%)$ were predicted to localize in the nucleus, whereas the remaining predicted $C p b H L H$ proteins were located in other organelles, including plastids, the cytoplasm, and chloroplasts. Additionally, some predicted $C p b H L H$ proteins existed in multiple cellular components. For example, CpbHLH013 was located in three cellular components: chloroplasts, part of the cytoplasm, and the nucleus, which may reflect its multiple functions in various biological processes. The metabolic processes involved the greatest number of putative $C p b H L H$ proteins $(47,87.0 \%)$. Biosynthetic processes and gene expression involved the second greatest number of putative $C p b H L H$ proteins (46, $85.2 \%$ ). In addition, $C p b H L H$ proteins could respond to stimulus, morphogenesis, cell differentiation, and developmental process.

\section{Expression analysis of bHLH superfamily genes under different abiotic stresses}

The bHLH proteins have been characterized functionally in many plants with a vital role in the regulation of diverse biological processes, but little is known about their role in papaya. To analyze the functions of $C p b H L H s$ responding to abiotic stresses, the expression profiles of 22 selected genes under salt, drought, ABA and cold stresses were investigated by using qRT-PCR (Table 2 and Fig. 7). The results showed that 4 (CpbHLH011, CpbHLHO22, CpbHLHO27 and CpbHLH056) of 22 CpbHLH mRNAs were increased, and 3 CpbHLH (CpbHLH02O, CpbHLH053 and CpbHLH062) mRNAs were reduced more than 2-fold in salt (200 mM Nacl) treated papaya seedlings. Under drought stress (25\% PEG), 8 (CpbHLH011, CpbHLH022, CpbHLH027, CpbHLH046, CpbHLH050, CpbHLH052, CpbHLH056 and CpbHLH068) of 22 CpbHLH mRNAs were upregulated, and 3 CpbHLH (CpbHLHO2O, CpbHLHO42 and CpbHLH053) mRNAs were downregulated more than 2-fold. Under ABA treatment $(100 \mu \mathrm{M}), 3$ (CpbHLH027, CpbHLH052 and CpbHLH056) of 22 CpbHLH mRNAs were upregulated, and 5 
362

363

364

365

366

367

368

369

370

371

372

373

374

375

376

377

378

379

380

381

382

383

384

385

386

387

388

389

390

391

392

393

394

395

396

397

398

399

400

CpbHLH (CpbHLH019, CpbHLH020, CpbHLH042, CpbHLH053 and CpbHLH062) mRNAs

were downregulated. Under cold stress $\left(4^{\circ} \mathrm{C}\right)$, there were $4 \mathrm{CpbHLH}$ genes $(\mathrm{CpbHLHO27,}$ CpbHLH035, CpbHLH056 and CpbHLH062) whose expression increased more than 1.5-fold, and 4 CpbHLH (CpbHLH046, CpbHLHO50, CpbHLHO52 and CpbHLHO68) mRNAs were reduced more than 2-fold.

Interestingly, a few transcripts of $C p b H L H$ responded to all or multiple stresses. For instance, CpbHLH056 was sensitive to all four stresses and was upregulated distinctly under the four stresses. The orthologue of CpbHLH056 in Arabidopsis is BEE1 (AtbHLH044) (Fig. 1), which has been functionally characterized in previous reports. At low temperatures, BEE1 is a positive regulator of flavonoid accumulation (Petridis et al. 2016), which is consistent with our results. In addition, BEE1, BEE2 and BEE3 are functionally redundant positive regulators of BR (brassinosteroid) signaling, but these transcripts are repressed by ABA (Friedrichsen et al. 2002). However, we found the transcription of $C p$ bHLH056 was notably upregulated (>10-fold) under ABA treatment. More interestingly, CpbHLH042, which is an orthologue of BEE2 (Fig.1), was distinctly repressed by ABA (approximately 4-fold). These results suggested that $C p b H L H 056$ and $C p$ bHLH042 may provide different functionalities compared to Arabidopsis. Additionally, CpbHLH027 was also upregulated distinctly under four stresses. In Arabidopsis, the orthologue of $C p b H L H 027$ is AtbHLH116 (ICE1) (Fig. 1), which can be induced by Nacl, ABA and cold stresses, playing an important role in the cold-responsive signaling pathway via an ABAindependent pathway (Chinnusamy et al. 2003). There are two orthologues of CpbHLH027 in rice, one ortholog is OsICE2/OsbHLH001, is induced by salt stress, and its overexpression can enhanced the tolerance to freezing and salt stress (Deng et al. 2017; Li et al. 2010). OsICE1/OsbHLH002 is another ortholog in rice, which is induced by chilling stress. OsbHLH002 can positively regulates cold signaling via targeting $O s \mathrm{TPP} 1$, which encodes a keyenzyme for trehalose biosynthesis (Zhang et al. 2017). These results implied $C p$ bHLH027 plays essential roles in abiotic stresses in papaya. In addition, the transcript of $C p b H L H 062$ was also increased under cold treatment, its orthologue is $A t b H L H 033 /$ ICE2, which involving the cold response and the ABA pathway (Fursova et al. 2009; Kurbidaeva et al. 2014), implying the CpbHLH062 may involved in the cold stress. CpbHLH053 was downregulated under salt, drought and ABA stresses. The orthologue of CpbHLH053 is AtbHLH129 (Fig. 1), which is a transcription repressor that negatively regulates the ABA response in Arabidopsis (Tian et al. 2015), implying CpbHLH053 may have the similar function with the AtbHLH129 in the process of ABA response.

We should also noticed a few $C p b H L H s$ that showed distinct increases or decreases in their mRNA levels under different treatments, and these $C p b H L H s$ ' orthologues have not been reported in previous studies. For instance, $C p b H L H 050$ is notably upregulated ( $>10$-fold) under PEG treatment, CpbHLH046 is upregulated by PEG treatment, but sharply down regulated under ABA and cold treatments, implying these genes may have additional functions than response to drought by regulating root development. CpbHLHO2O and CpbHLHO53 were downregulated 
401 (>2-fold) by Nacl, PEG and ABA stresses distinctly. We should also pay attention to these genes 402 in the following research.

403

404

405

406

407

408

409

410

411

412

413

414

415

416

417

418

419

420

421

422

423

424

425

426

427

428

429

430

431

432

433

434

435

436

437

438

439

440

\section{Discussion}

Transcription factors (TFs) play key roles in the stress regulation network and signal pathways in plants. Basic/helix-loop-helix (bHLH) TFs are the second largest TFs family in plants and have been identified in many species (Cheng et al. 2018; Gao et al. 2017; Geng \& Liu 2018; Mao et al. 2017; Sun et al. 2015; Toledo-Ortizet al. 2003; Wang et al. 2018a; Wang et al. 2018b; Zhang et al. 2018). However, the bHLH TF family has not previously been reported in papaya (Carica papaya $L$.). In this paper, we found $73 \mathrm{CpbHLH}$ genes in papaya. This TF family seemed to be one of the moderately sized families compared with other plant species, which might be because of the papaya has a relatively small reference genome, the size is only $372 \mathrm{Mb}$ (Ray et al. 2008). The gene evolution changes the gene organization. In this study, we found that CpbHLH genes were diverse in their number introns, ranging from 0 to 10 (Data S2). This result implied these genes may have undergone numerous of genetic evolution events, and the genes in different subfamilies may have different functions (Cheng et al. 2018). Most CpbHLHs in the same subfamily shared similar gene structures and protein motifs according to the analysis of exon/intron organizations and motif compositions (Fig. 2, 3 and 4), indicating that the functions of encoded proteins in each subfamily are probably stable. However, the conserved motif analysis showed that some $C p b H L H s$, which are mainly distributed in eight subfamilies (including $\mathrm{V}$ a, V b, IIIf, IVa, IIIb, III, I b and I a subfamilies) from the phylogenetic tree, harbor another conserved motif (motif 3 ) with a length of 36 amino acids (Fig. 2 and 4), indicating that these proteins may have additional functions.

Promoter cis-acting regulatory element analysis showed that cis-elements could be divided into three main categories: light responsive, abiotic and biotic stresses and plant growth and development. Especially in abiotic and biotic stresses, the most common response elements were ABA (29.15\%), MeJA (23.62\%) and SA (9.78\%), suggesting that these phytohormones may play important roles in the regulation of papaya growth and development (Fig. 5 and Data S4). In addition, the promoter cis-acting element involved in the abscisic acid responsiveness analysis is consistent with the qRT-PCR results (Data S4 and Table 2), showing four genes (CpHLH020/027/-053/-056) involved in abscisic acid response. Another two genes (CpHLH020/-062) were also identified that were involved in abscisic acid response by GO annotation analysis and qRTPCR (Data S6 and Table 2). We also identified a large number of cis-acting elements in CpbHLH genes that may respond to drought (MBS, 8.67\%), which is also consistent with the qRT-PCR (including seven genes: CpbHLH027/-050/-056/-011/-068/-042/-053). Other genes also had important elements, including LTR, TC-rich and WUN-motifs, which indicated plant responses to low temperatures, defense stresses and wound-responsiveness, respectively. These results implied $C p b H L H$ genes may have a wide range of functions in papaya growth, disease resistance, and response to environmental conditions. We also analyze the cis-elements in the promoter regions of putative orthologous genes in Arabidopsis (Fig. S1 and Data S4). And we 
441 compared the promoter cis-elements of bHLH genes in Arabidopsis, papaya and previously

442 reported bamboo (Cheng et al. 2018). The result showed that the promoter cis-elements of $b H L H$

443 genes in these three plants were divided into three categories, and most of elements were the

444 same. These similar results implied that most of the promoter cis-elements of bHLH family were

445 conserved in Arabidopsis, papaya and bamboo. The most notable is, the percentage of MeJA

446 responsive cis-elements in papaya and Arabidopsis $(23.62 \%, 30.61 \%)$ were less than bamboo

$447(43.39 \%)$. And the percentage of SA responsive cis-elements in papaya and Arabidopsis $(9.78 \%$,

$4485.87 \%$ ) were also less than bamboo (10.31\%). SA is a phytohormone that plays important roles

449 in plant defenses against pathogens (Pokotylo et al. 2019). MeJA also has been identified as a

450 vital cellular regulator that mediates defense processes (Cheong \& Choi 2003). So, the stress-

451 responsive elements in papaya and Arabidopsis were corresponding less than bamboo, including

452 drought-responsive elements, wound-responsive elements, low temperature-responsive elements,

453 and defense and stress responsive elements. These results may help explain why papaya is more

454 sensitive to external stresses compared to bamboo.

455 Many studies have shown that $b H L H$ genes are involved in various abiotic and biotic stresses

456 responses. We randomly selected 22 genes to investigate their expression profiles by using qRT-

457 PCR under salt, drought, ABA and cold stresses (Fig.7 and Table 2). The results revealed some

458 candidate $C p b H L H$ genes that might be responsible for abiotic stress responses in papaya. For

459 example, CpbHLH027, CpbHLH062, AtbHLH116 (ICE1), AtbHLH33 (ICE2), OsbHLH001

460 (OsICE2) and OsbHLH002 (OsICE1) were clustered within one clade. Among them,

461 AtbHLH116 (ICE1), AtbHLH33 (ICE2), OsbHLH001 (OsICE2) and OsbHLH002 (OsICE1)

462 have been reported function in chilling stress in Arabidopsis and rice (Chinnusamy et al. 2003;

463 Deng et al. 2017; Fursova et al. 2009; Li et al. 2010; Zhang et al. 2017). And the transcripts of

464 CpbHLH027, CpbHLH062 were increased under chilling stress in this study, implying that

465 CpbHLH027 and CpbHLH062 may be also involved in the process of chilling stress. The

466 orthologue of CPHLH056 in Arabidopsis is BEE1 (At1G18400), which is a positive regulator of

467 flavonoid accumulation (Petridis et al. 2016). BEE1, BEE2 and BEE3 are functionally redundant

468 positive regulators of BR signaling, and their transcription is repressed by ABA in Arabidopsis

469 (Friedrichsen et al. 2002). However, we found that the transcription of CpbHLHO56 is notably

470 upregulated ( $>10$-fold) under ABA treatment rather than downregulated in this study. These

471 results imply that CpbHLH056 may involved in the process of ABA stress but has different

472 function compared to Arabidopsis. We have also noticed a few candidate CpbHLHs that showed

473 distinct increases or decreases in their mRNA levels under different treatments, and these

474 CpbHLHs' orthologues have not been reported in other plants. For instance, CpbHLH050,

475 CpbHLH020, CpbHLH046, CpbHLH053, and so on. These findings provide important candidate

476 genes/proteins necessary for further functional research on the bHLH family in papaya.

477

478 Conclusions

479 In conclusion, the study performed a genome-wide analysis of basic helix-loop-helix (bHLH)

480 transcription factors in papaya. As a result, a total of $73 \mathrm{bHLH}$ genes were identified in papaya, 
481

482

483

484

485

486

487

488

489

490

491

492

493

494

495

496

497

498

499

500

501

502

503

504

505

506

507

508

509

510

511

512

513

514

515

516

517

518

519

520

and these CpbHLHs were classified into 18 subfamilies with one orphan, which was consistent with the earlier results showing that the bHLH subfamily in plants can be divided into 15-25 subfamilies. Almost all of the CpbHLHs in the same subfamily shared similar gene structures and protein motifs according to analysis of exon/intron organizations and motif compositions. These results further supported the classification predicted by the phylogenetic tree. Compared to rice and Arabidopsis, the amino acid sequences of the $C p b H L H$ domains were quite conservative, especially Leu-27 and Leu-63. Promoter cis-element and GO annotation analysis revealed that most of the CpbHLHs could respond to various biotic/abiotic stress-related events. Abiotic stress treatment and quantitative real-time PCR (qRT-PCR) assay further supported promoter $c i s$-acting regulatory element and GO annotation analysis, revealed some important candidate $\mathrm{CpbHLHs}$ that might be responsible for abiotic stress responses in papaya. We completed the first comprehensive genome-wide analysis of the $b H L H$ gene family in papaya, and our results provide information necessary for further functional research of the bHLH family in papaya.

\section{Figure Titles and Legends}

Figure 1: Phylogenic and family members analysis of bHLHs from papaya, rice and Arabidopsis.

(A) The 73 CpbHLHs are clustered into 18 subfamilies. Phylogenetic tree was constructed based on the neighbor-joining method. Bootstrapping with 1000 replicates was used to assess the statistical reliability of nodes in the tree. (B) Comparison of bHLH family members from papaya, rice and Arabidopsis. Different colors represent the different plants. Green: OsbHLHs, red: AtbHLHs, Blue: CpbHLHs.

\section{Figure 2: Gene structure and motif distribution of the papaya bHLH family.}

(A) Exon-intron organization of $C p b H L H$ genes. Exons and introns are presented as filled orange sticks and thin black single lines, respectively. The brackets and Roman numerals separate each subfamily and clearly present the member conservation of each subfamily. (B) Arrangements of conserved motifs in $73 \mathrm{CpbHLH}$ proteins. Three predicted motifs are represented by different colored boxes, motif 1 (red block), motif 2 (blue block) and motif 3 (green block).

\section{Figure 3: Multiple sequence alignment of the bHLH domains in papaya.}

Amino acids with more than 50\% identity are labeled with colored boxes. Figure 4: Motif composition and logos of papaya bHLH proteins.
(A) The logos of motif 1 and 2, which together constitute the bHLH domain in papaya. The overall height of the character represents the conservation of an amino acid at the specific position. Each color of the English letters represents a type of amino acid residue. (B) The logo of motif 3, which is another conserved motif.

Figure 5: Cis-acting element analysis of the promoter of $\mathrm{bHLH}$ genes in papaya.
(A) Percentage of total cis-acting elements in the promoter region of $C p b H L H$ genes. (B) The percentage of each cis-acting element in the abiotic and biotic stresses categories.

Figure 6: Gene ontology (GO) annotation of $C p b H L H$ proteins. 
521 The annotation was performed on three categories, (A) molecular function, (B) biological

522 processes and (C) cellular components.

523 Figure 7: Quantitative RT-PCR analysis of 22 selected $C \mathrm{pbHLH}$ genes under cold stress 524 condition $\left(4^{\circ} \mathrm{C}\right)$.

525 The data are expressed as means \pm SD of three independent biological determinations. Untreated

526 seedlings were used as the control groups. ${ }^{*} \mathrm{P}<0.05$ and $* * \mathrm{P}<0.01$ (Student's $\mathrm{t}$ test) indicate

527 significant differences between treated seedlings and control groups.

528

529

530

\section{Supplemental Information}

531 Supplemental Data S2: Detailed information of CpbHLH genes and CpbHLH proteins.

532 Supplemental Data S3: bHLH subfamily members of papaya, rice and Arabidopsis.

533 Supplemental Data S4: Promoter analysis of bHLH genes in papaya and Arabidopsis.

534 Supplemental Data S5 and Supplemental Data S6: GO annotation of $C p b H L H$ proteins.

535 Table S1: The information of 32 predicted proteins from the PlantTFDBv2.0 that were excluded 536 by Interproscan and SMART.

537 Table S2: Consensus sequences of bHLH domains in papaya, rice and Arabidopsis.

538 Supplemental file 1: Raw data of qRT-PCR assay.

539 Figure S1: Cis-acting element analysis of the promoter of bHLH genes in Arabidopsis.

540 (A) Percentage of total cis-acting elements in the promoter region of AtbHLH genes. (B) The

541 percentage of each cis-acting element in the abiotic and biotic stresses categories.

542

543 Additional Information and Declarations

544 Authors' contributions

545 Min yang conceived and designed the experiments, performed the experiments, analyzed the

546 data, prepared figures and/or tables, authored or reviewed drafts of the paper, approved the final

547 draft.

548 Chenping Zhou and $\mathrm{Hu}$ Yang performed the experiments, analyzed the data, prepared figures

549 and/or tables, authored or reviewed drafts of the paper, approved the final draft.

550 Ruibin Kuang and Bingxiong Huang performed the experiments, analyzed the data, authored or 551 reviewed drafts of the paper, approved the final draft.

552 Yuerong Wei conceived and designed the experiments, authored or reviewed drafts of the paper, 553 approved the final draft.

554 Data Availability

555 Putative bHLH transcription factors were downloaded from the PlantTFDBv2.0

556 (http://planttfdb.cbi.pku.edu.cn/).The genomic sequences, ID numbers and coding sequences

557 (CDS) corresponding to each predicted $b H L H$ gene were obtained from the Phytozome database 558 (https://phytozome.jgi.doe.gov/pz/portal.html).The online tool Multiple EM for Motif Elicitation 559 (MEME, version 5.02) was used to search for conserved motifs among the bHLH proteins 
560 (http://meme-suite.org/tools/meme) by uploading the protein sequences of the papaya bHLH 561 superfamily.

562

563

564

565

566

567

568

569

570

571

572

573

574

575

576

577

578

579

580

581

582

583

584

585

586

587

588

589

590

591

592

593

594

595

596

597

598

599

600

601

602

603

\section{References.}

Agarwal PK, Agarwal P, Reddy MK, and Sopory SK. 2006. Role of DREB transcription factors in abiotic and biotic stress tolerance in plants. Plant Cell Rep 25:1263-1274.

Amoutzias GD, Robertson DL, and Bornberg-Bauer E. 2004. The evolution of protein interaction networks in regulatory proteins. Comp Funct Genomics 5:79-84.

Atchley WR, Fitch WM. 1997. A natural classification of the basic helix-loop-helix class of transcription factors. Proceedings of the National Academy of Sciences 94:5172-5176.

Atchley WR, Terhalle W, and Dress A. 1999. Positional Dependence, Cliques, and Predictive Motifs in the bHLH Protein Domain. Journal of Molecular Evolution 48:501-516.

Bailey TL, and Elkan C. 1994. Fitting a mixture model by expectation maximization to discover motifs in biopolymers. p 28-36.

Bernhardt C, Lee MM, Gonzalez A, Zhang F, Lloyd A, and Schiefelbein J. 2003. The bHLH genes GLABRA3 (GL3) and ENHANCER OF GLABRA3 (EGL3) specify epidermal cell fate in the Arabidopsis root. Development 130:6431-6439.

Buck MJ, and Atchley WR. 2003. Phylogenetic analysis of plant basic helix-loop-helix proteins. $J$ Mol Evol 56:742-750.

Carretero-Paulet L, Galstyan A, Roig-Villanova I, Martinez-Garcia JF, Bilbao-Castro JR, and Robertson DL. 2010. Genome-wide classification and evolutionary analysis of the bHLH family of transcription factors in Arabidopsis, poplar, rice, moss, and algae. Plant Physiol 153:1398-1412.

Chen YY, Li MY, Wu XJ, Huang Y, Ma J, and Xiong AS. 2015. Genome-wide analysis of basic helix-loop-helix family transcription factors and their role in responses to abiotic stress in carrot. Molecular Breeding 35:125.

Cheng X, Xiong R, Liu H, Wu M, Chen F, Hanwei Y, and Xiang Y. 2018. Basic helix-loop-helix gene family: Genome wide identification, phylogeny, and expression in Moso bamboo. Plant Physiol Biochem 132:104-119.

Cheong J-J, and Choi YD. 2003. Methyl jasmonate as a vital substance in plants. Trends in Genetics 19:409-413.

Chinnusamy V, Ohta M, Kanrar S, Lee BH, Hong X, Agarwal M, and Zhu JK. 2003. ICE1: a regulator of cold-induced transcriptome and freezing tolerance in Arabidopsis. Genes Dev 17:1043-1054.

Deng C, Ye H, Fan M, Pu T, and Yan J. 2017. The rice transcription factors OsICE confer enhanced cold tolerance in transgenic Arabidopsis. Plant Signal Behav 12:e1316442.

Farquharson KL. 2016. A Domain in the bHLH Transcription Factor DYT1 Is Critical for Anther Development. Plant Cell 28:997-998.

Feller A, Machemer K, Braun EL, and Grotewold E. 2011. Evolutionary and comparative analysis of MYB and bHLH plant transcription factors. Plant $J$ 66:94-116.

Feng Y, Xu P, Li B, Li P, Wen X, An F, Gong Y, Xin Y, Zhu Z, Wang Y, and Guo H. 2017. Ethylene promotes root hair growth through coordinated EIN3/EIL1 and RHD6/RSL1 activity in Arabidopsis. Proceedings of the National Academy of Sciences of the United States of America 114:13834-13839.

Peer) reviewing PDF | (2020:02:45453:1:0:CHECK 15 Apr 2020) 
604 Friedrichsen DM, NemhauserJ, Muramitsu T, Maloof JN, Alonso J, Ecker JR, Furuya M, and

605

606

607

608

609

610

611

612

613

614

615

616

617

618

619

620

621

622

623

624

625

626

627

628

629

630

631

632

633

634

635

636

637

638

639

640

641

642

643

644

645

646

647
Chory J. 2002. Three redundant brassinosteroid early response genes encode putative bHLH transcription factors required for normal growth. Genetics, 162:1445-1456.

Fursova OV, Pogorelko GV, and Tarasov VA. 2009. Identification of ICE2, a gene involved in cold acclimation which determines freezing tolerance in Arabidopsis thaliana. Gene 429:98-103.

Gao C, Sun J, Wang C, Dong Y, Xiao S, Wang X, and Jiao Z. 2017. Genome-wide analysis of basic/helix-loop-helix gene family in peanut and assessment of its roles in pod development. PLoS One 12:e0181843.

Geng J, and Liu JH. 2018. The transcription factor CsbHLH18 of sweet orange functions in modulation of cold tolerance and homeostasis of reactive oxygen species by regulating the antioxidant gene. J Exp Bot 69:2677-2692.

Guo XJ, and Wang JR. 2017. Global identification, structural analysis and expression characterization of bHLH transcription factors in wheat. BMC Plant Biol 17:90.

Heim MA, Jakoby M, Werber M, Martin C, Weisshaar B, and Bailey PC. 2003. The basic helixloop-helix transcription factor family in plants: a genome-wide study of protein structure and functional diversity. Mol Biol Evol 20:735-747.

Hu B, Jin J, Guo AY, Zhang H, Luo J, and Gao G. 2015. GSDS 2.0: an upgraded gene feature visualization server. Bioinformatics 31:1296-1297.

Jiang Y, Yang B, and Deyholos MK. 2009. Functional characterization of the Arabidopsis bHLH92 transcription factor in abiotic stress. Mol Genet Genomics 282:503-516.

Jin J, Tian F, Yang DC, Meng YQ, Kong L, Luo J, and Gao G. 2017. PlantTFDB 4.0: toward a central hub for transcription factors and regulatory interactions in plants. Nucleic Acids Res 45:D1040-D1045.

Jones S. 2004. An overview of the basic helix-loop-helix proteins. Genome Biology 5:226.

Kaotoh MK, Kuma K, and Miyata T. 2002. MAFFT: a novel method for rapid multiple sequence alignment based on fast Fourier transform. Nucleic Acids Resarch 30: 3059-3066.

Kosugi S, and Ohashi Y. 2002. DNA binding and dimerization specificity and potential targets for the TCP protein family. The Plant Journal 30:337.

Kurbidaeva A, Ezhova T, and Novokreshchenova M. 2014. Arabidopsis thaliana ICE2 gene: phylogeny, structural evolution and functional diversification from ICE1. Plant Sci 229:10-22.

Ledent V, and Vervoort M. 2001. The Basic Helix-Loop-Helix Protein Family: Comparative Genomics and Phylogenetic Analysis. Genome Res 11:754-770.

Lee S, Lee S, Yang KY, Kim YM, Park SY, Kim SY, and Soh MS. 2006. Overexpression of PRE1 and its homologous genes activates Gibberellin-dependent responses in Arabidopsis thaliana. Plant Cell Physiol 47:591-600.

Leivar P, Monte E, Al-Sady B, Carle C, Storer A, Alonso JM, Ecker JR, and Quail PH. 2008. The Arabidopsis phytochrome-interacting factor PIF7, together with PIF3 and PIF4, regulates responses to prolonged red light by modulating phyB levels. Plant Cell 20:337-352.

Li F, Guo S, Zhao Y, Chen D, Chong K, and Xu Y. 2010. Overexpression of a homopeptide repeat-containing bHLH protein gene (OrbHLH001) from Dongxiang Wild Rice confers freezing and salt tolerance in transgenic Arabidopsis. Plant Cell Rep 29:977-986.

Li X, Duan X, Jiang H, Sun Y, Tang Y, Yuan Z, Guo J, Liang W, Chen L, Yin J, Ma H, Wang J, 
648

649

650

651

652

653

654

655

656

657

658

659

660

661

662

663

664

665

666

667

668

669

670

671

672

673

674

675

676

677

678

679

680

681

682

683

684

685

686

687

688

689

690

691

692

693 and Zhang D. 2006. Genome-wide analysis of basic/helix-loop-helix transcription factor family in rice and Arabidopsis. Plant Physiol 141:1167-1184.

Liu K, Yuan C, Feng S, Zhong S, Li H, Zhong J, Shen C, and Liu J. 2017a. Genome-wide analysis and characterization of Aux/IAA family genes related to fruit ripening in papaya (Carica papaya L.). BMC Genomics 18:351.

Liu K, Yuan C, Li H, Lin W, Yang Y, Shen C, and Zheng X. 2015. Genome-wide identification and characterization of auxin response factor (ARF) family genes related to flower and fruit development in papaya (Carica papaya L.). BMC Genomics 16:901.

Liu W, Tai H, Li S, Gao W, Zhao M, Xie C, and Li WX. 2014. bHLH122 is important for drought and osmotic stress resistance in Arabidopsis and in the repression of ABA catabolism. New Phytol 201:1192-1204.

Liu Y, Li J, Wei G, Sun Y, Lu Y, Lan H, Li C, Zhang S, and Cao M. 2017. Cloning, molecular evolution and functional characterization of ZmbHLH16, the maize ortholog of OsTIP2 (OsbHLH142). Biol Open 6:1654-1663.

Magali L, Patrice D, Gert T, Kathleen M, Yves M, Yves VdP, Pierre R, and Stephane R. 2002. PlantCARE, a database of plant cis-acting regulatory elements and a portal to tools for in silico analysis of promoter sequences. Nucleic Acids Res 30:325-327.

Mao K, Dong Q, Li C, Liu C, and Ma F. 2017. Genome Wide Identification and Characterization of Apple bHLH Transcription Factors and Expression Analysis in Response to Drought and Salt Stress. Front Plant Sci 8:480.

Ming R, Hou S, Feng Y, Yu Q, Dionne-Laporte A, Saw JH, Senin P, Wang W, Ly BV, Lewis KL, et al. 2008. The draft genome of the transgenic tropical fruit tree papaya (Carica papaya Linnaeus). Nature 452:991-996.

Morgenstern B, and Atchley WR. 1999. Evolution of bHLH transcription factors: modular evolution by domain shuffling? Molecular Biology \& Evolution 16:1654-1663.

Murre C, Mccaw PS, and Baltimore D. 1989. A new DNA binding and dimerization motif in immunoglobulin enhancer binding, daughterless, MyoD, and myc proteins. Cell 56:777783.

Niu X, Guan Y, Chen S, and Li H. 2017. Genome-wide analysis of basic helix-loop-helix (bHLH) transcription factors in Brachypodium distachyon. BMC Genomics 18:619.

Pan LJ, and Jiang L. 2014. Identification and expression of the WRKY transcription factors of Carica papaya in response to abiotic and biotic stresses. Mol Biol Rep 41:1215-1225.

Peraza-Echeverria S, Santamaría JM, Fuentes G, de los Ángeles Menéndez-Cerón M, VallejoReyna MÁ, and Herrera-Valencia VA. 2012. The NPR1 family of transcription cofactors in papaya: insights into its structure, phylogeny and expression. Genes \& Genomics 34:379-390.

Petridis A, Döll S, Nichelmann L, Bilger W, and Mock HP. 2016. Arabidopsis thaliana G2-LIKE FLAVONOID REGULATOR and BRASSINOSTEROID ENHANCED EXPRESSION1 are low-temperature regulators of flavonoid accumulation. New Phytologist 211:912-925.

Pires N, and Dolan L. 2010. Origin and diversification of basic-helix-loop-helix proteins in plants. Mol Biol Evol 27:862-874.

Pokotylo I, Kravets V, and Ruelland E. 2019. Salicylic Acid Binding Proteins (SABPs): The Hidden Forefront of Salicylic Acid Signalling. International Journal of Molecular Sciences 20:4377.

Porter BW, Paidi M, Ming R, Alam M, Nishijima WT, and Zhu YJ. 2009. Genome-wide analysis of Carica papaya reveals a small NBS resistance gene family. Mol Genet Genomics

Peer) reviewing PDF | (2020:02:45453:1:0:CHECK 15 Apr 2020) 
694

695

696

697

698

699

700

701

702

703

704

705

706

707

708

709

710

711

712

713

714

715

716

717

718

719

720

721

722

723

724

725

726

727

728

729

730

731

732

733

734

735

736

737

738

281:609-626.

Price MN, Dehal PS, and Arkin AP. 2009. FastTree: computing large minimum evolution trees with profiles instead of a distance matrix. Mol Biol Evol 26:1641-1650.

Ray M, Shaobin H, Yun F, Qingyi Y, Alexandre DL, Saw JH, Pavel S, Wei W, Ly BV, and Lewis $\mathrm{KLT}$. 2008. The draft genome of the transgenic tropical fruit tree papaya (Carica papaya Linnaeus). Nature 452:991.

Roig-Villanova I, Bou-Torrent J, Galstyan A, Carretero-Paulet L, Portolés S, RodríguezConcepción M, and Martínez-García JF. 2007. Interaction of shade avoidance and auxin responses: a role for two novel atypical bHLH proteins. EMBO JOURNAL 26:4756-4767.

Simionato E, Ledent V, Richards G, Thomas-Chollier M, Kerner P, Coornaert D, Degnan BM, and Vervoort M. 2007. Origin and diversification of the basic helix-loop-helix gene family in metazoans: insights from comparative genomics. BMC Evol Biol 7:33.

Song Y, Liu H-d, Zhou Q, Zhang H-j, Zhang Z-d, Li Y-d, Wang H-b, and Liu F-z. 2017. Highthroughput sequencing of highbush blueberry transcriptome and analysis of basic helixloop-helix transcription factors. Journal of Integrative Agriculture 16:591-604.

Eddy SR. 1998. Profile hidden Markov models. Bioinformatics 14: 755-763.

Stevens JD, Roalson EH, and Skinner MK. 2008. Phylogenetic and expression analysis of the basic helix-loop-helix transcription factor gene family: genomic approach to cellular differentiation. Differentiation 76:1006-1022.

Sun $\mathrm{H}$, Fan $\mathrm{HJ}$, and Ling HQ. 2015. Genome-wide identification and characterization of the bHLH gene family in tomato. BMC Genomics 16:9.

Tian H, Guo H, Dai X, Cheng Y, Zheng K, Wang X, and Wang S. 2015. An ABA down-regulated bHLH transcription repressor gene, bHLH129 regulates root elongation and ABA response when overexpressed in Arabidopsis. Sci Rep 5:17587.

Tian T, Liu Y, Yan H, You Q, Yi X, Du Z, Xu W, and Su Z. 2017. agriGO v2.0: a GO analysis toolkit for the agricultural community, 2017 update. Nucleic Acids Res 45:W122-W129.

Toledo-Ortiz G, Huq E, and Quail PH. 2003. The Arabidopsis Basic/Helix-Loop-Helix Transcription Factor Family. The Plant Cell Online 15:1749-1770.

Vervoort M, and Ledent V. 2001. The evolution of the neural basic Helix-Loop-Helix proteins. ScientificWorldJournal 1:396-426.

Wang P, Su L, Gao H, Jiang X, Wu X, Li Y, Zhang Q, Wang Y, and Ren F. 2018a. GenomeWide Characterization of bHLH Genes in Grape and Analysis of their Potential Relevance to Abiotic Stress Tolerance and Secondary Metabolite Biosynthesis. Front Plant Sci 9:64.

Wang R, Zhao P, Kong N, Lu R, Pei Y, Huang C, Ma H, and Chen Q. 2018b. Genome-Wide Identification and Characterization of the Potato bHLH Transcription Factor Family. Genes (Basel) 9.

Wu S, and Zhu Y. 2012. ProPAS: standalone software to analyze protein properties. Bioinformation 8:167-169.

Xu G, Guo C, Shan H, and Kong H. 2012. Divergence of duplicate genes in exon-intron structure. Proc Natl Acad Sci U S A 109:1187-1192.

$\mathrm{Xu} \mathrm{Y,} \mathrm{Xu} \mathrm{H,} \mathrm{Wall} \mathrm{MM,} \mathrm{and} \mathrm{Yang} \mathrm{J.} \mathrm{2020.} \mathrm{Roles} \mathrm{of} \mathrm{transcription} \mathrm{factor} \mathrm{SQUAMOSA} \mathrm{promoter}$ binding protein-like gene family in papaya (Carica papaya) development and ripening. Genomics.

Zhang C, Feng R, Ma R, Shen Z, Cai Z, Song Z, Peng B, and Yu M. 2018. Genome-wide

Peer] reviewing PDF | (2020:02:45453:1:0:CHECK 15 Apr 2020) 
739 analysis of basic helix-loop-helix superfamily members in peach. PLoS One $740 \quad$ 13:e0195974.

741 Zhang JZ, Creelman RA, and Zhu J-K. 2004. From Laboratory to Field. Using Information from

742

743

744

745

746

747

748

749 Arabidopsis to Engineer Salt, Cold, and Drought Tolerance in Crops. Plant Physiol 135:615-621.

Zhang Z, Li J, Li F, Liu H, Yang W, Chong K, and Xu Y. 2017. OsMAPK3 Phosphorylates OsbHLH002/OsICE1 and Inhibits Its Ubiquitination to Activate OsTPP1 and Enhances Rice Chilling Tolerance. Developmental Cell 43:731-743.e735.

Zhu E, You C, Wang S, Cui J, Niu B, Wang Y, Qi J, Ma H, and Chang F. 2015. The DYT1interacting proteins bHLH010, bHLH089 and bHLH091 are redundantly required for Arabidopsis anther development and transcriptome. The Plant Journal 83:976-990.

750

751

752

Zhu X, Li X, Chen W, Chen J, Lu W, Chen L, and Fu D. 2012. Evaluation of new reference genes in papaya for accurate transcript normalization under different experimental conditions. PLoS One 7:e44405.

753 


\section{Figure 1}

Phylogenic and family members analysis of bHLHs from papaya, rice and Arabidopsis.

(A) The $73 \mathrm{CpbHLHs}$ are clustered into 18 subfamilies. Phylogenetic tree was constructed based on the neighbor-joining method. Bootstrapping with 1000 replicates was used to assess the statistical reliability of nodes in the tree. (B) Comparison of bHLH family members from papaya, rice and Arabidopsis. Different colors represent the different plants. Green: OsbHLHs, red: AtbHLHs, Blue: CpbHLHs. 
A.

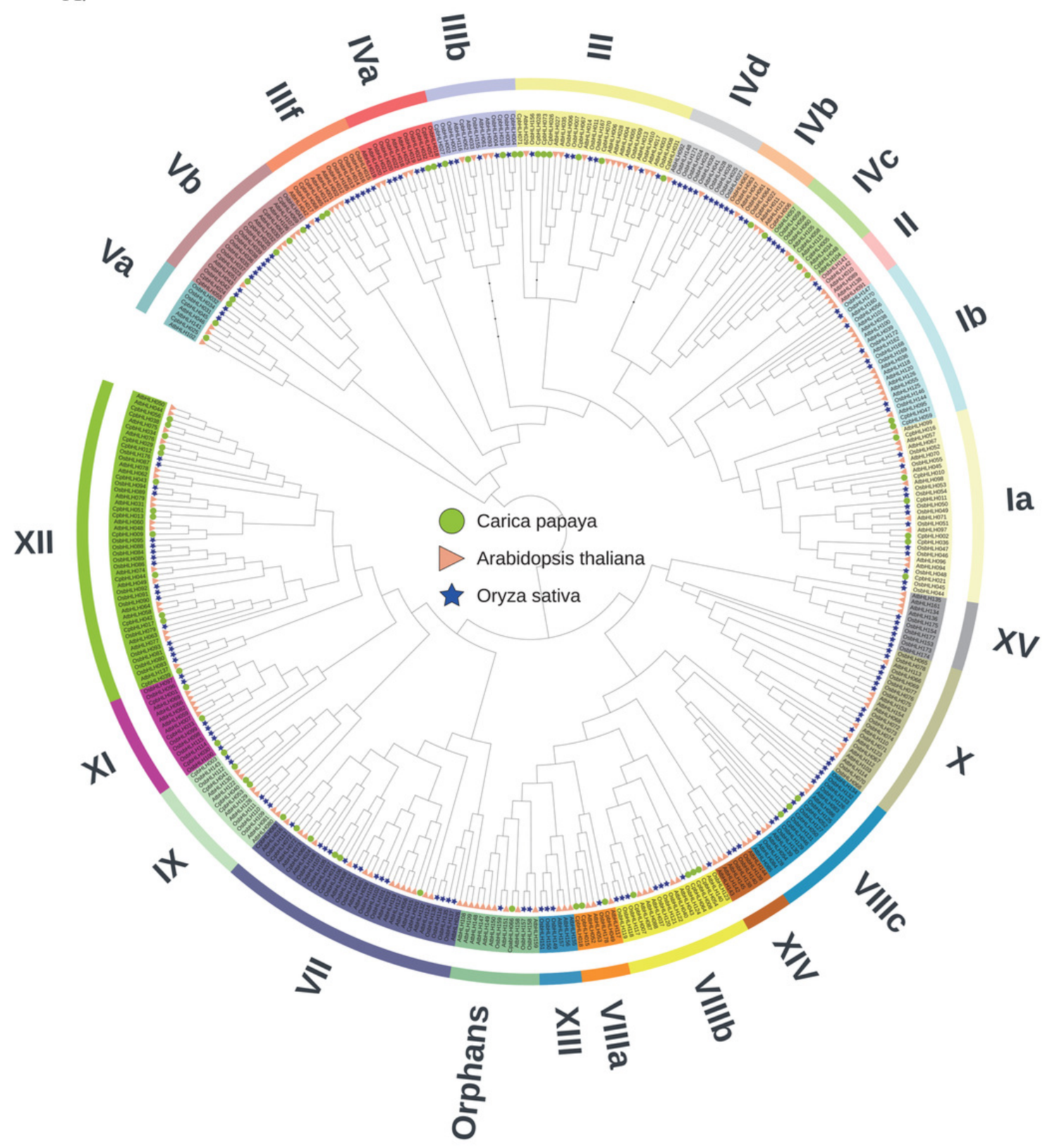

B.

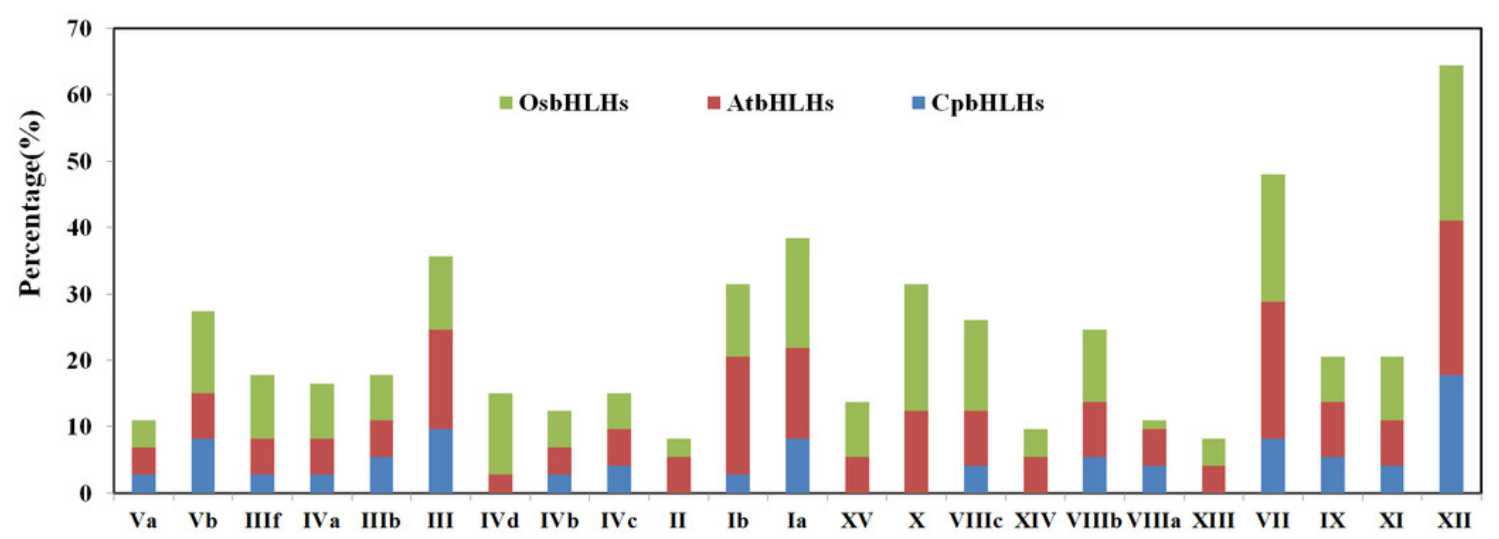




\section{Figure 2}

Gene structure and motif distribution of the papaya bHLH family.

(A) Exon-intron organization of $\mathrm{CpbHLH}$ genes. Exons and introns are presented as filled orange sticks and thin black single lines, respectively. The brackets and Roman numerals separate each subfamily and clearly present the member conservation of each subfamily. (B) Arrangements of conserved motifs in $73 \mathrm{CpbHLH}$ proteins. Three predicted motifs are represented by different colored boxes, motif 1 (red block), motif 2 (blue block) and motif 3 (green block). 
A.

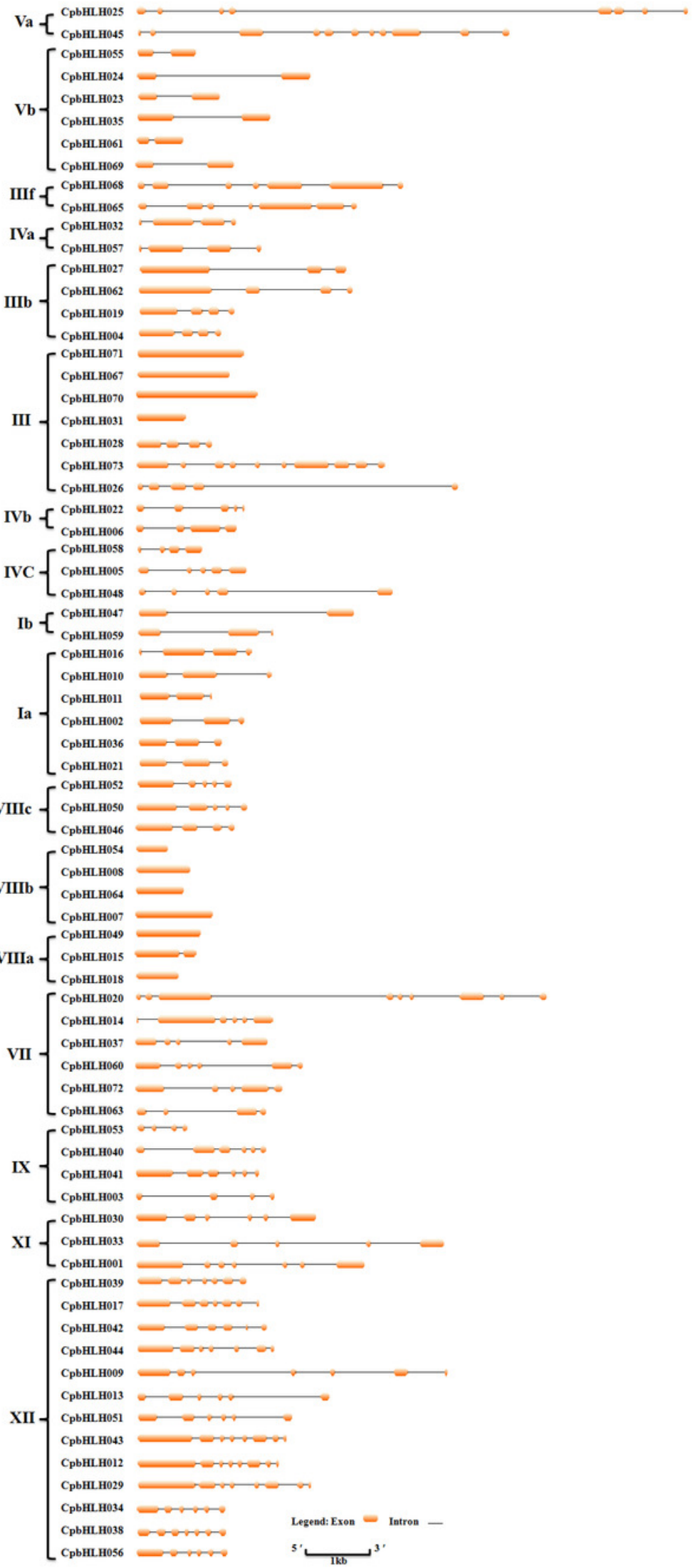

B.

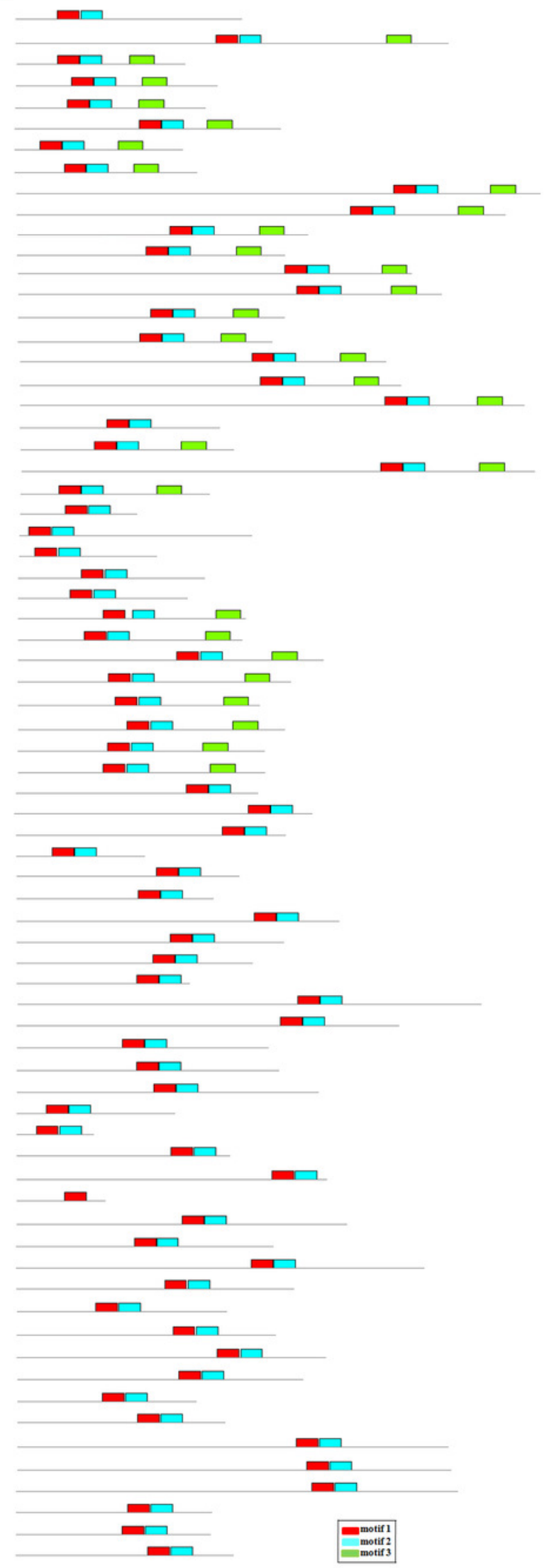


Figure 3

Multiple sequence alignment of the bHLH domains in papaya.

Amino acids with more than $50 \%$ identity are labeled with colored boxes. 


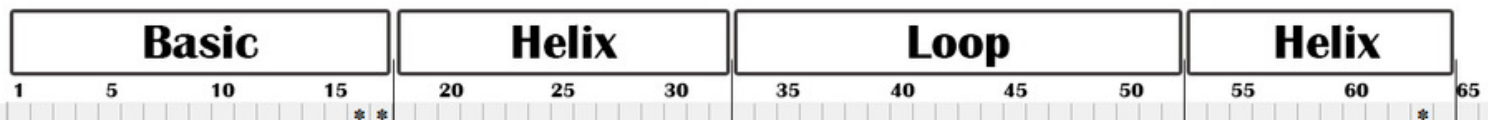

Species/Abbrv 1. CpbHLH010 2. CpbHLH011

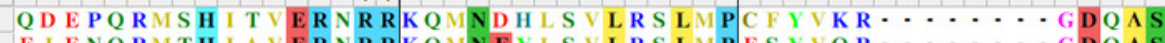
E I ENQRMTH I A VERRRKQMNEYIS V LRS LMPESYVOR........ GDOAS 4. CpbHLH002 E I E NORMT H I E ENQRMTH 5. CpbHLH021 6. CpbHLH016 7. CpbHLHO01 8. CpbHLH030 9. CpbHLH003 10. CpbHLH033 10. CpbHLH033 11. CpbHLH053 13. CpbHLH04 14. CpbHLH034 15. CpbHLH056 16. CpbHLH038 17. CpbHLHO09 18. CpbHLH039 19. CpbHLH043 20. CpbHLH044 21. CpbHLH029 22. CpbHLH012 23. CpbHLH042 24. CpbHLH017 25. CpbHLH013 26. CpbHLH051 27. CpbHLH027 28. CpbHLH067 29. CpbHLH071 30. CpbHLH031 31. CpbHLH070 32. CpbHLH068 33. CpbHLH02. 34. CpbHLH050 35. CpbHLH046 36. CpbHLH052 37. CpbHLHOO8 38. CpbHLHO64 39. CpbHLHO07 40. CpbHLH015 41. CpbHLH018 42. CpbHLH055 43. CpbHLH025 44. CpbHLH045 45. CpbHLH061 46. CpbHLH024 47. CpbHLH035 48. CpbHLHO69 49. CpbHLH048 50. CpbHLH005 51. CpbHLH058 52. CpbHLHOO6 53. CpbHLH022 54. CpbHLH065 54. CpbHLH065
55. CpbHLH047 56. CpbHLH059 57. CpbHLHOOA 58. CpbHLH019 59. CpbHLH026 60. CpbHLHO66 61. CpbHLH062 62. CpbHLH028 63. CpbHLH073 64. CpbHLH054 65. CpbHLH049 66. CpbHLH072 67. CpbHLH063 68. CpbHLH014 69. CpbHLH037 70. CpbHLH020 71. CpbHLH060 Q V E N Q R M T H E V ES Q R M T H RRGQATD PHS I A R R R R R MND HLNS LR S L M P P S Y I Q R RRGQA TDPHS I A ER LR R ER I A ERMKA LQE L V P N A - . N . RRGOATDPHS I A ER RRER I ERMKALOE LV P RRGQATDPHS I A ERLRREK I A ERIRALQELVP

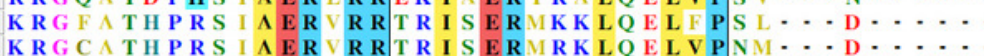
KRGCATHPRS I A ERVRRTR ISSERMRKLQ ELVP NM・ - D -

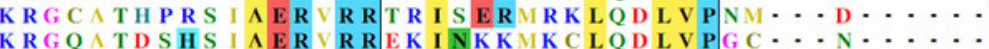
R RG

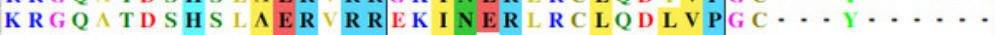
RRGQA TDSHSL A ERARREK I NARMKLIQ E L V P G C . . N . . . . R R G Q A T D S H S L A E R V R R E K I S ERMK I L Q K L V P G C . . D . . . . . RRGQ A TDSHSL A E R V R R E K I S E RMKL L Q D L V P G C R R G Q A T N S H S L A E R V R R E K I S ERMR L L Q E L V P G C . . N . . . . . . R R G Q A T N H S L A E R VR R EK I S E RMK F L Q D L V P G C . . S . . . . R R G Q A T D S H S L A ER VR REK I S ERMK F L Q D L V P G C . . N . . . . . RRG Q A T D S H S L A ER R R R EK I S KKMK C L Q D L V P G C . . N . . . .

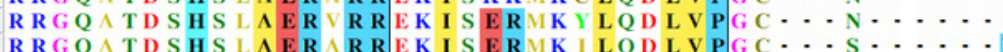

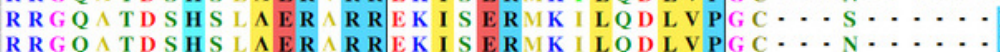
K R K GM P A K N L M A ERR R RKK L NDR L Y M L R S V PK I . . . . . S K GR - ET P L NHVEA ERORREKLNHRF Y L R V V P N . . . . . S S . G C S D S P L NHVEA ERQRRERLNNRF Y A R S V V P N . . . . . . S K

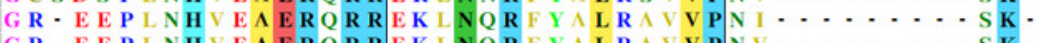

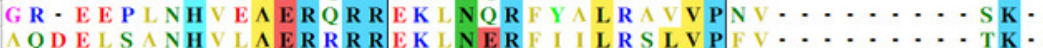
KA A S V S K S H S Q A EKRR RDR I NA Q L A T LRK L I P K S . . D . . . . K .

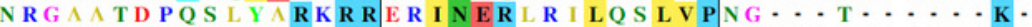
SRG TA T D PQS L Y A RKRRER I N ER LK I L Q N L V P N G . T . . . . .

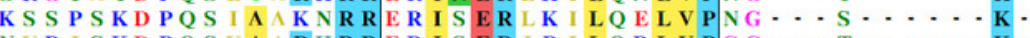

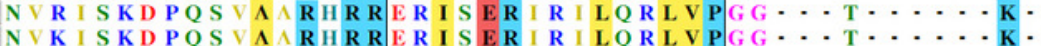

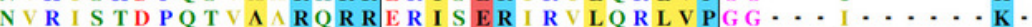

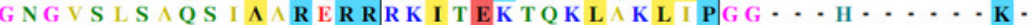

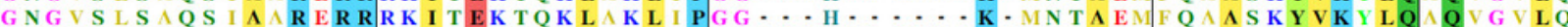

TAEM Q A A S KYVKYLO OVG L L

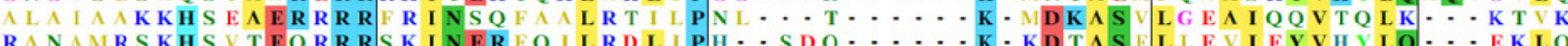

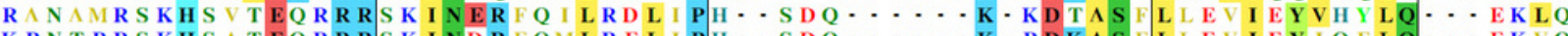

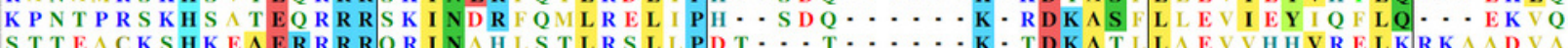
年

KALA L K S H S E A ERRR RER I NA H L D T L R G L V P S T . -

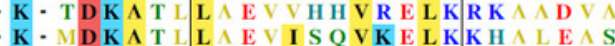
K A LA A S K S H S E A ERRR R E R I N N H L A K L R S L L P S T - . T . . . K - T D K A S I

R L L A L K N HK E A EKR R RER I NS H L N K L R T L L P C N - S . . . . K - T D K A S L L A K V Q R V K E L K Q Q T N E I S

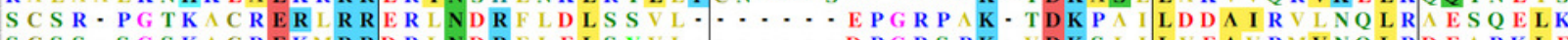

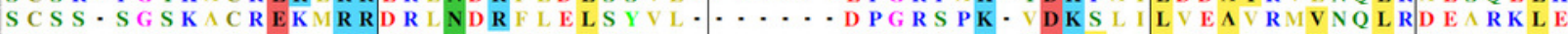

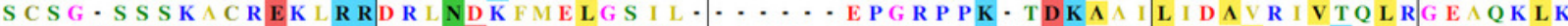

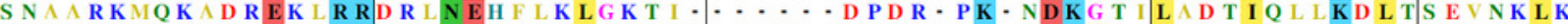

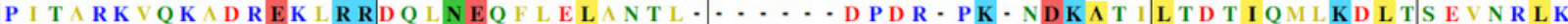

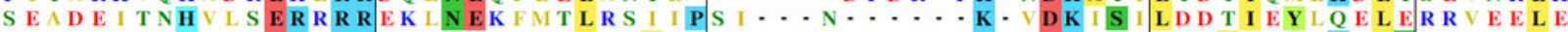

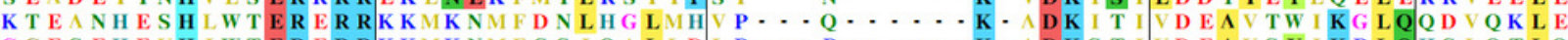

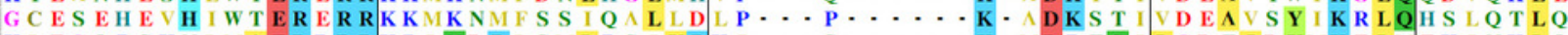
K L E G Q P S K N L M A ER R R RKR L NDRL S MLR S I V P K I . - S . . . K K KM E G Q P S K N LM A ER R R RK R L N D R I S M L R S I V P N I . . S . . . K .

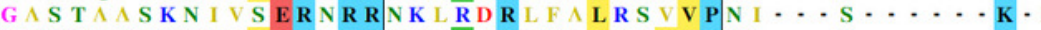

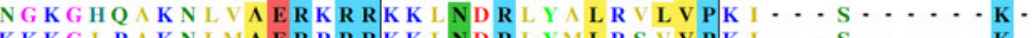

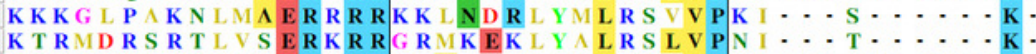
GS E T Y Q S K N L V T E R N R R N R I K D G L R A I V P

G V K L S T D P Q S V A A R E R R H R I S D R F K I L Q S M V P G G * . T . . . . K .

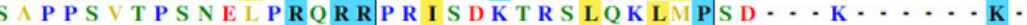
R N I RA A A TH N E S ER R R RD R I NQKMK T L Q K L V P N A . S . . . . K .

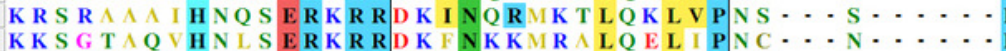
KR T R S A E L H N I S ERRR R D R I KGKM R A L Q E L I P N C . . N . . . . . K R S R A E V H N L S ERR R R D R I N E KM RA L Q E L I P R C K R S RA A E V H N L S EKRR R S R I N EKMK A L Q N L I P N S . . N . . . . K 72. CpbHLH057

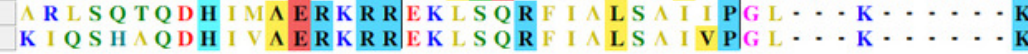

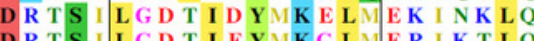

D R T S I L G D T I E Y M K G L M E R I K T L Q

D K A S I I K D A I D Y I O E L H E O E R R I Q D K A S I L G D A I E Y V Q E L Q K Q A K E L Q D R A S I L L G D A I D Y L K E L L Q R I S D L H D K A S I V G D A V L Y V E D L Q M Q K K L K

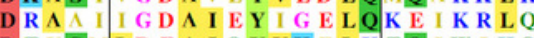

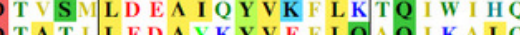
LED A Y K Y V E F L Q A O I K A L Q T D K A S M L D E V I E Y L T K Q L K - VDKA AM L D E A I E Y M K T L Q L Q L Q M MW K - L D R A S M L D D A I N Y L K A L K L Q V E M L S K- S D K A S M L D E A I E Y L K S L Q L Q V Q M I S K - MD K A S L L G D A I K Y I KO L O E R K K I L E 73. CpbHLH032

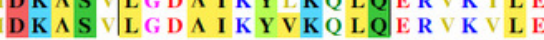


Figure 4

Motif composition and logos of papaya bHLH proteins.

(A) The logos of motif 1 and 2, which together constitute the bHLH domain in papaya. The overall height of the character represents the conservation of an amino acid at the specific position. Each color of the English letters represents a type of amino acid residue. (B) The logo of motif 3, which is another conserved motif.

A. Logo of motif 1

Logo of motif 2

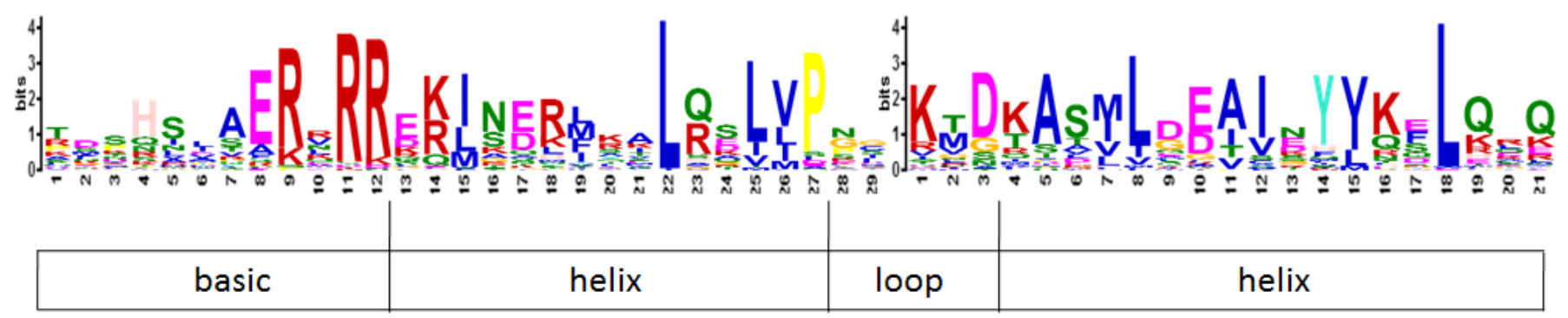

B.

Logo of motif 3

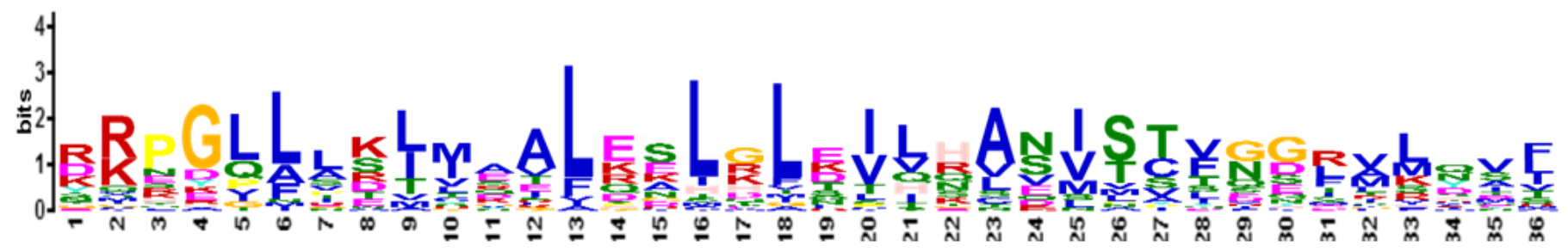




\section{Figure 5}

Cis-acting element analysis of the promoter of $b H L H$ genes in papaya.

(A) Percentage of total cis-acting elements in the promoter region of $\mathrm{CpbHLH}$ genes. (B) The percentage of each cis-acting element in the abiotic and biotic stresses categories.

A.

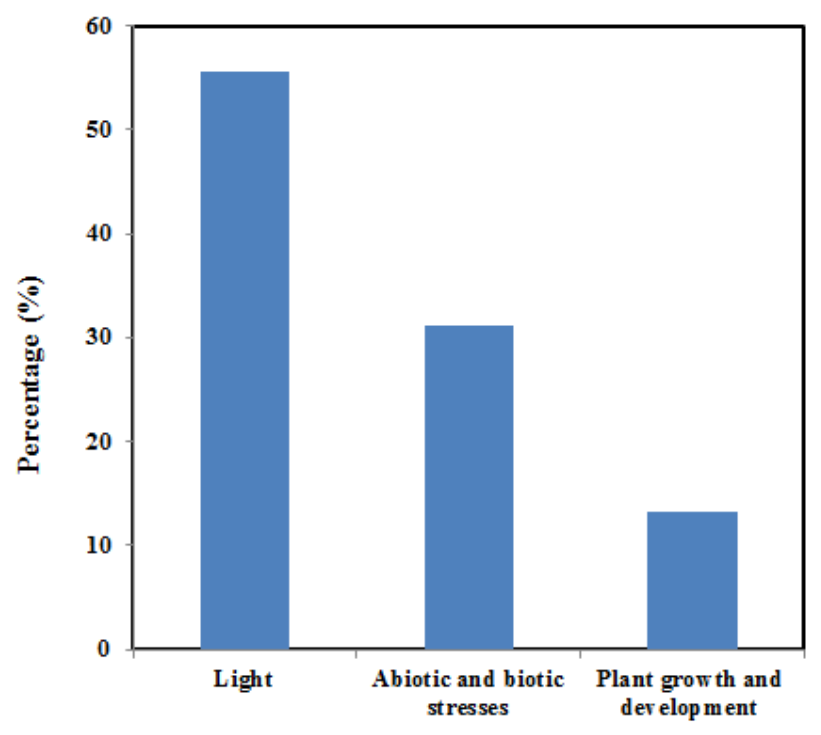

B.

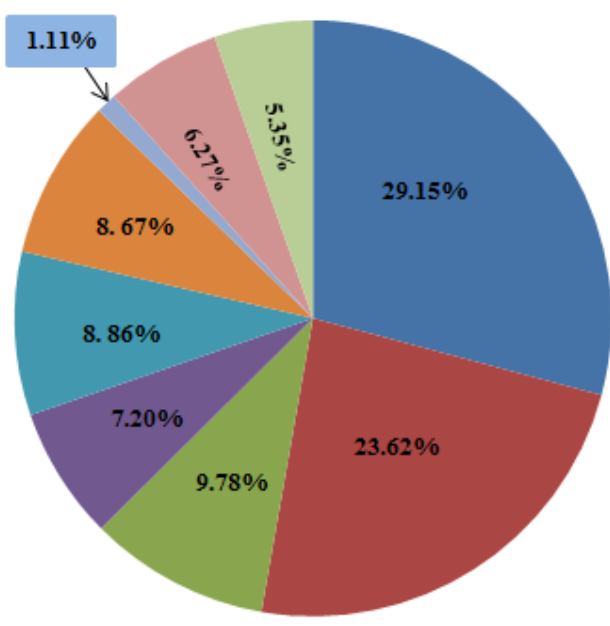

Abscisic acid

M ethyl jasmonate

Salicylic acid

Auxin

n Gibb erellin

Drought

Wound

Defense and stress

Low-temperature 
Figure 6

Gene ontology (GO) annotation of CpbHLH proteins.

The annotation was performed on three categories, (A) molecular function, (B) biological processes and (C) cellular components.

A.

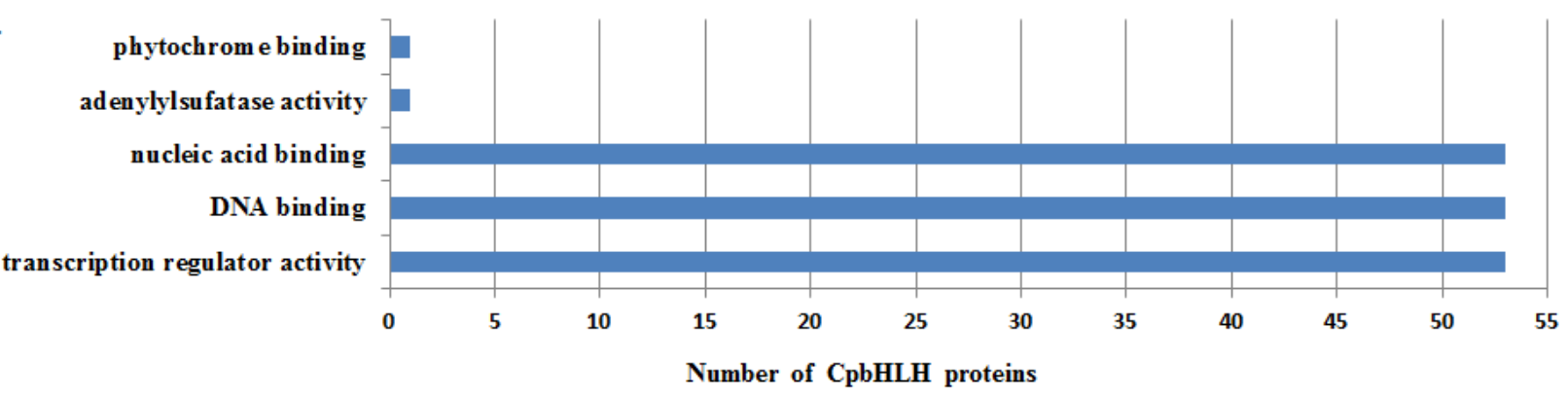

B.

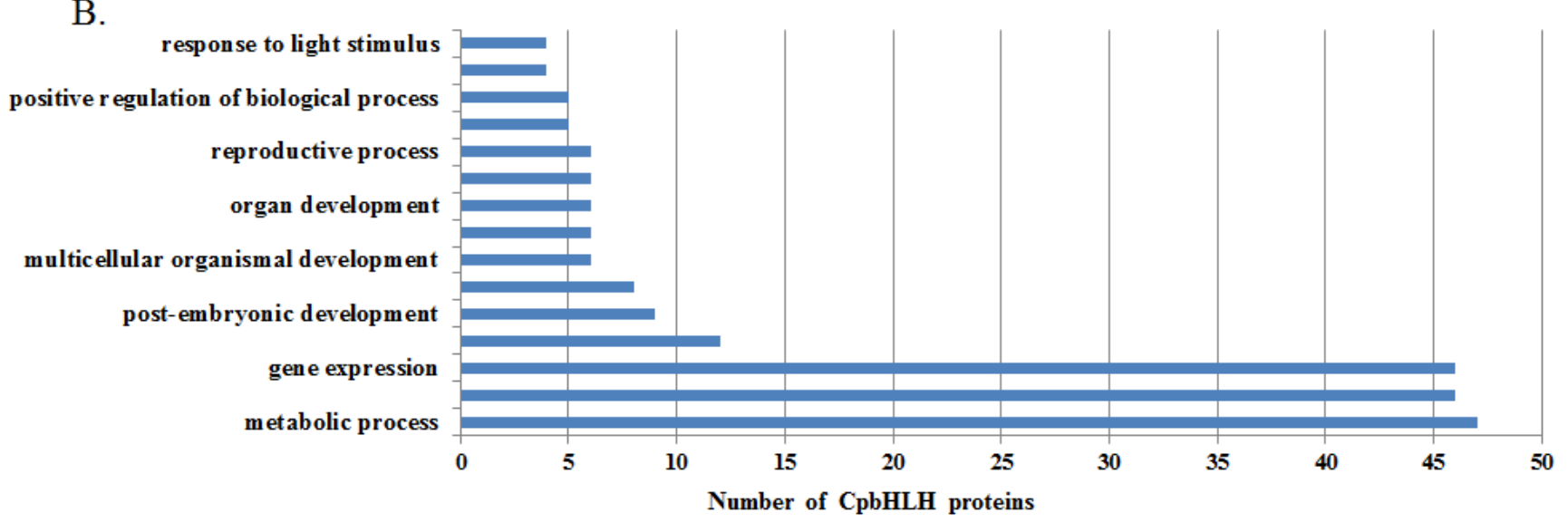

C.

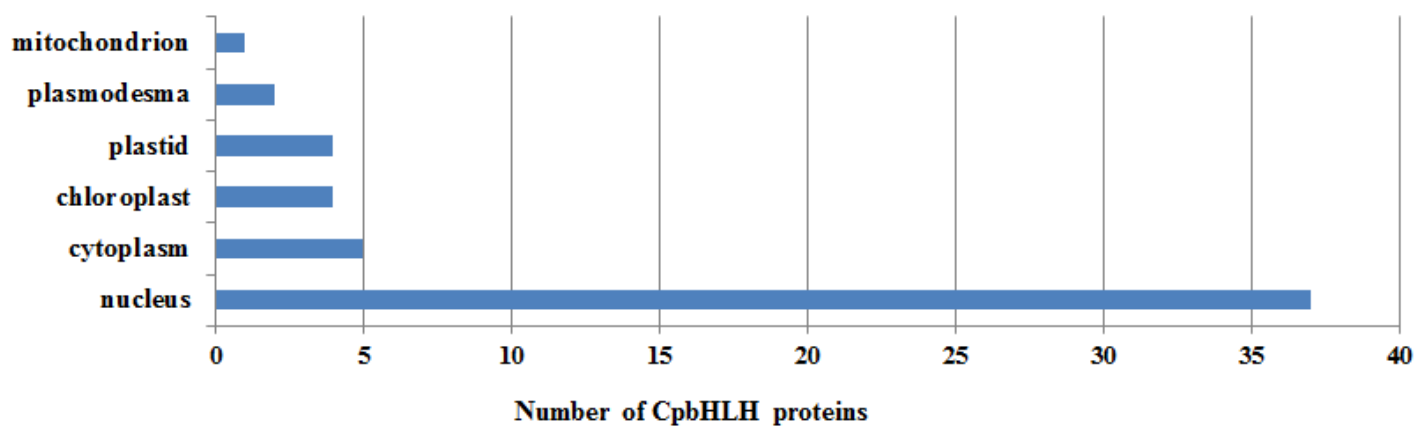


Figure 7

Quantitative RT-PCR analysis of 22 selected $\mathrm{CPbHLH}$ genes under cold stress condition $\left(4^{\circ} \mathrm{C}\right)$.

The data are expressed as means \pm SD of three independent biological determinations.

Untreated seedlings were used as the control groups. ${ }^{*} \mathrm{P}<0.05$ and ${ }^{* * P}<0.01$ (Student's $\mathrm{t}$ test) indicate significant differences between treated seedlings and control groups.

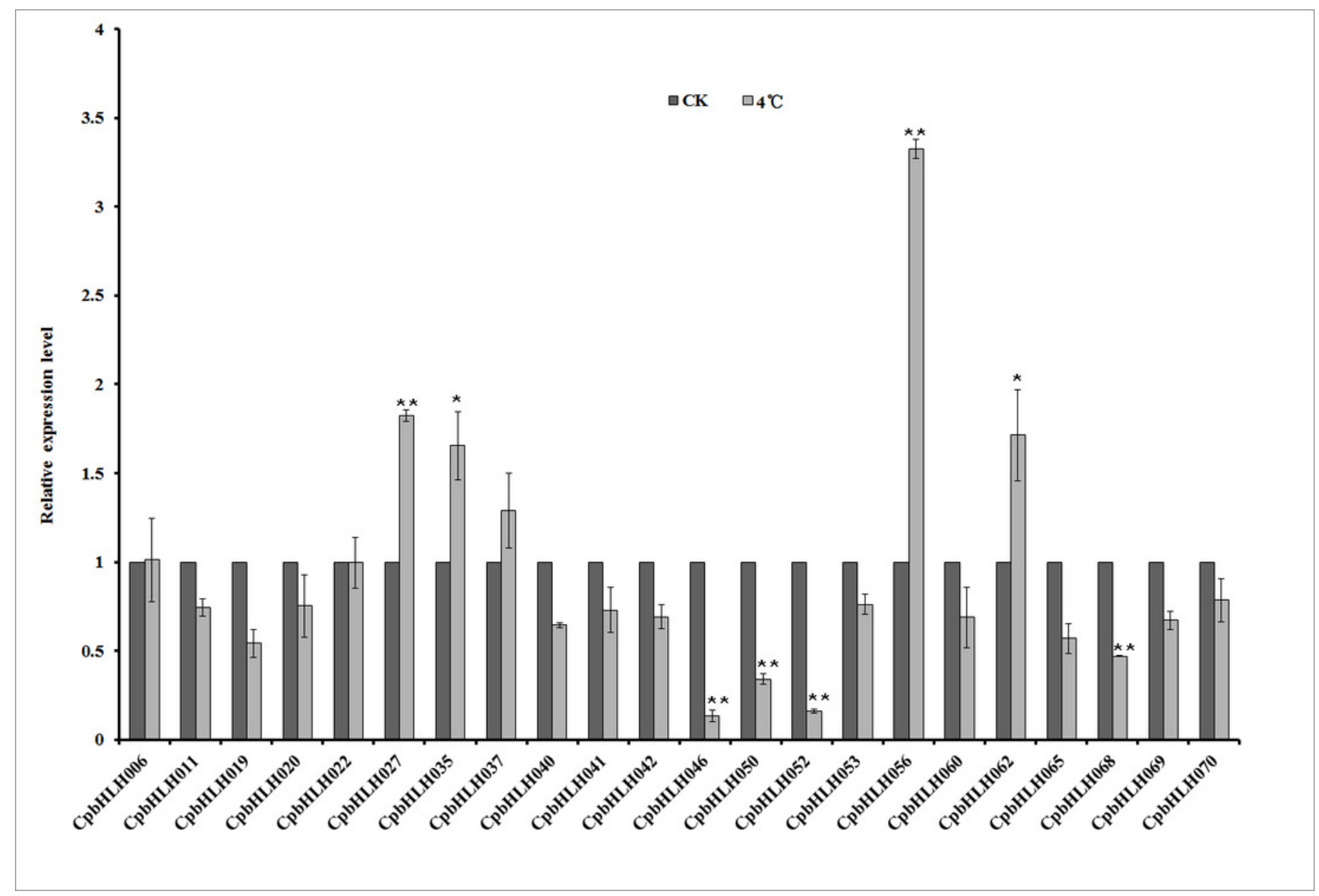




\section{Table $\mathbf{1}$ (on next page)}

Summary of TFs identified from dicotyledonous plant species with genome sequences 
1 Table 1:

2 Summary of TFs identified from dicotyledonous plant species with genome sequences

\begin{tabular}{lllll}
\hline Plant species & Common name & bHLH & Proteins & Ratio (\%) \\
\hline C. papaya & Papaya & 73 & 27829 & 0.26 \\
A. ipaensis/A. duranensis & Peanut & $129 / 132$ & 7243 & $1.78 / 1.82$ \\
Malus $x$ domestica & Apple & 188 & 15173 & 1.24 \\
S. lycopersicum & Tomato & 159 & 15722 & 1.01 \\
A. thaliana & Arabidopsis & 147 & 32125 & 0.46 \\
S. tuberosum & Potato & 124 & 17445 & 0.71 \\
P. persica & Peach & 95 & 28299 & 0.34 \\
V. vinifera & Wine Grapes & 94 & 47097 & 0.20 \\
C. sinensis & Valencia Orange & 56 & 13522 & 0.41 \\
\hline
\end{tabular}

3 


\section{Table 2 (on next page)}

Expression levels of $\mathrm{CPbHLH}$ genes under salt, drought and ABA stresses

Quantitative RT-PCR was used to investigate the expression levels (shown in fold change) of the CpbHLHs. The expression level in the control (CK) was set at 1.0. The means of three replicates of qRT-PCR and standard deviations (SD) values are shown. 
1 Table 2:

2 Expression levels of $\mathrm{CpbHLH}$ genes under salt, drought and $\mathrm{ABA}$ stresses

\begin{tabular}{|c|c|c|c|c|}
\hline The name of $C p b H L H s$ & $\mathbf{C K}$ & $\mathrm{NaCl}(200 \mathrm{mM})$ & PEG (25\%) & $\mathrm{ABA}(100 \mu \mathrm{M})$ \\
\hline СрbHLH006 & 1 & $0.74 \pm 0.13$ & $0.95 \pm 0.15$ & $0.75 \pm 0.07$ \\
\hline СрbHLH011 & 1 & $2.45 \pm 0.04$ & $4.06 \pm 0.12$ & $1.66 \pm 0.12$ \\
\hline СрbHLH019 & 1 & $0.80 \pm 0.03$ & $0.64 \pm 0.02$ & $0.18 \pm 0.02$ \\
\hline CpbHLH020 & 1 & $0.20 \pm 0.003$ & $0.39 \pm 0.01$ & $0.22 \pm 0.01$ \\
\hline СрbHLHO22 & 1 & $4.21 \pm 0.03$ & $3.05 \pm 0.03$ & $1.54 \pm 0.08$ \\
\hline CpbHLH027 & 1 & $4.49 \pm 0.09$ & $2.32 \pm 0.06$ & $2.27 \pm 0.12$ \\
\hline Cр bHLH035 & 1 & $1.62 \pm 0.08$ & $1.79 \pm 0.07$ & $0.71 \pm 0.02$ \\
\hline Cр bHLH037 & 1 & $0.96 \pm 0.02$ & $1.04 \pm 0.01$ & $1.92 \pm 0.07$ \\
\hline СрbHLH040 & 1 & $1.38 \pm 0.04$ & $0.77 \pm 0.03$ & $0.51 \pm 0.03$ \\
\hline Cр $b H L H 041$ & 1 & $0.55 \pm 0.01$ & $0.76 \pm 0.03$ & $0.70 \pm 0.03$ \\
\hline СpbHLH042 & 1 & $0.55 \pm 0.03$ & $0.33 \pm 0.04$ & $0.26 \pm 0.03$ \\
\hline СpbHLH046 & 1 & $0.78 \pm 0.06$ & $5.46 \pm 0.10$ & $0.53 \pm 0.03$ \\
\hline CpbHLH050 & 1 & $0.53 \pm 0.04$ & $11.86 \pm 0.10$ & $1.18 \pm 0.02$ \\
\hline CpbHLH052 & 1 & $1.64 \pm 0.06$ & $8.71 \pm 0.06$ & $4.42 \pm 0.13$ \\
\hline CpbHLH053 & 1 & $0.44 \pm 0.04$ & $0.31 \pm 0.02$ & $0.19 \pm 0.01$ \\
\hline CpbHLH056 & 1 & $7.11 \pm 0.04$ & $32.65 \pm 0.45$ & $12.22 \pm 0.21$ \\
\hline Ср $b H L H 060$ & 1 & $0.89 \pm 0.07$ & $0.76 \pm 0.05$ & $0.75 \pm 0.11$ \\
\hline Cp $b H L H 062$ & 1 & $0.38 \pm 0.02$ & $0.65 \pm 0.02$ & $0.30 \pm 0.02$ \\
\hline СpbHLH065 & 1 & $1.64 \pm 0.06$ & $1.04 \pm 0.03$ & $0.52 \pm 0.05$ \\
\hline CpbHLH068 & 1 & $1.72 \pm 0.05$ & $2.37 \pm 0.04$ & $1.39 \pm 0.07$ \\
\hline СрbHLH069 & 1 & $0.68 \pm 0.07$ & $0.89 \pm 0.18$ & $0.52 \pm 0.08$ \\
\hline CpbHLH070 & 1 & $1.11 \pm 0.02$ & $1.17 \pm 0.07$ & $1.47 \pm 0.04$ \\
\hline
\end{tabular}

3 Quantitative RT-PCR was used to investigate the expression levels (shown in fold change) of the CpbHLHs. The expression level

4 in the control (CK) was set at 1.0. The means of three replicates of qRT-PCR and standard deviations (SD) values are shown. 